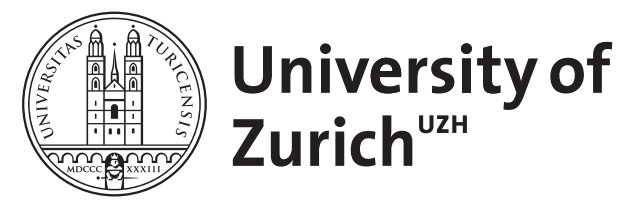

\title{
Finite number density corrections to leptogenesis
}

\author{
Beneke, M ; Garbrecht, B ; Herranen, M ; Schwaller, P
}

\begin{abstract}
We derive and solve kinetic equations for leptogenesis within the Closed Time Path (CTP) formalism. It is particularly emphasised how the procedure of real intermediate state subtraction familiar from the Boltzmann approach is realised within the CTP framework; and we show how in timeindependent situations no lepton asymmetry emerges, in accordance with the CPT theorem. The CTP approach provides new quantum statistical corrections from evaluating the loop integrals. These lead to an enhancement of the asymmetry originating from the Bose statistics of the Higgs particles. To quantify this effect, we define and evaluate an effective CP-violating parameter. We also solve the kinetic equations and show explicitly that the new quantum statistical corrections can be neglected in the strong-washout regime, while, depending on initial conditions, they can be sizable for weak washout.
\end{abstract}

DOI: https://doi.org/10.1016/j.nuclphysb.2010.05.003

Posted at the Zurich Open Repository and Archive, University of Zurich ZORA URL: https://doi.org/10.5167/uzh-46939

Journal Article

Accepted Version

Originally published at:

Beneke, M; Garbrecht, B; Herranen, M; Schwaller, P (2010). Finite number density corrections to leptogenesis. Nuclear Physics. Section B, 838(1-2):1-27.

DOI: https://doi.org/10.1016/j.nuclphysb.2010.05.003 
TTK-10-16

ZU-TH 02/10

1002.1326 [hep-ph]

February 5, 2010

\title{
Finite Number Density Corrections to Leptogenesis
}

\author{
Martin Beneke, Björn Garbrecht, Matti Herranen \\ Institut für Theoretische Teilchenphysik und Kosmologie, \\ RWTH Aachen University, \\ D-52056 Aachen, Germany \\ and \\ Pedro Schwaller \\ Institut für Theoretische Physik, \\ Universität Zürich, CH-8057 Zürich, Switzerland
}

\begin{abstract}
We derive and solve kinetic equations for leptogenesis within the Closed Time Path (CTP) formalism. It is particularly emphasised how the procedure of real intermediate state subtraction familiar from the Boltzmann approach is realised within the CTP framework; and we show how in time-independent situations no lepton asymmetry emerges, in accordance with the CPT theorem. The CTP approach provides new quantum statistical corrections from evaluating the loop integrals. These lead to an enhancement of the asymmetry originating from the Bose statistics of the Higgs particles. To quantify this effect, we define and evaluate an effective $C P$-violating parameter. We also solve the kinetic equations and show explicitly that the new quantum statistical corrections can be neglected in the strong washout regime, while, depending on initial conditions, they can be sizable for weak washout.
\end{abstract}




\section{Introduction}

In the light of the present knowledge of elementary particle physics phenomenology, leptogenesis appears as one of the most plausible explanations for the dynamical emergence of the baryon asymmetry of the Universe $[1,2]$. Leptogenesis in turn is a variant of baryogenesis from out-of-equilibrium decays of heavy particles during the expansion and cooling of the early Universe [3, 4]. The deviation from equilibrium provides the breaking of time reversal invariance, that is a necessary condition for baryogenesis [5] as a consequence of the CPT theorem.

The most common approach for making quantitative predictions is to formulate and solve a network of Boltzmann equations, that describes the evolution of the number densities of the particle species relevant to baryogenesis. In simple scenarios for leptogenesis, a setup that leads to meaningful results is to compute the distribution function of a heavy right-handed Majorana neutrino $N_{1}$ and of the charge density of a lepton doublet $\ell$, while one may assume that the remaining species are in thermal equilibrium. More realistic models typically take account of the presence of the several generations. The Boltzmann equations are usually written in a form, where on the left hand side, there is a kinetic term that traces the particle number distributions, while on the right hand side, there is a collision term. The collision term accounts for elastic and inelastic (particle number violating) scatterings. It arises from weighting reaction rates, computed in vacuum scattering theory (in-out formalism), with the distribution functions of the species involved.

When setting up the Boltzmann equations, one encounters the following issue: Let $\Gamma_{N i \rightarrow \ell \phi}$, the rate for the decay process $N_{i} \rightarrow \ell \phi$, be proportional to $1+\varepsilon$, where $\varepsilon$ parametrises the $C P$ asymmetry of the decay. Then, by complex conjugation of the involved coupling constants, it follows that $\Gamma_{N i \rightarrow \bar{\ell} \phi^{*}} \propto 1-\varepsilon$. Application of the $C P T$ theorem then implies that the inverse decay rates $\Gamma_{\ell \phi \rightarrow N i} \propto 1-\varepsilon$ and $\Gamma_{\bar{\ell} \phi^{*} \rightarrow N i} \propto 1+\varepsilon$. If these were the only contributions that are accounted for in the collision term, one might conclude that the two-by-two rate $\ell \phi \rightarrow \bar{\ell} \phi^{*}$ is proportional to $1-2 \varepsilon$, while the rate of $\bar{\ell} \phi^{*} \rightarrow \ell \phi$ is proportional to $1+2 \varepsilon$. An asymmetry even in equilibrium would result, in apparent contradiction with CPT invariance. But of course, there is no contradiction, since the heavy neutrinos are unstable and therefore do not belong to the Hilbert space of asymptotic states of a unitary $S$-matrix. The total lepton number violating twoby-two rates are therefore not given by the above naive multiplication of the rates for decay and inverse decay. Yet, it is crucial that one tracks the neutrino densities in the Boltzmann equations. The issue is fixed when realising that the resonant parts of the two-by-two scatterings, where the intermediate neutrino is on-shell, contribute to the Boltzmann equations at the same order in coupling constants as the decay and inverse decay processes. Technically, one adds the full two-by-two rates and subtracts from these the on-shell contribution, since this is already accounted for in the decays and inverse decays [4]. This procedure is known as the subtraction of real intermediate states (RIS).

One expects that the RIS subtraction is naturally realised within a first principle approach, that is less heuristic than the Boltzmann ansatz. This first principle approach 
is given by applying the Schwinger-Keldysh Closed Time Path (CTP) formalism [6, 7] to field theory [8]. In this paper, we derive and solve the Kadanoff-Baym equations for leptogenesis, that result from the CTP formalism. We pay particular attention to demonstrating that no lepton asymmetry is generated in equilibrium, or, more generally in situations that are time-reversal invariant. For models with fermionic leptons this result has been reported earlier in works based on the CTP formalism $[9,10,11]$, though no detailed derivation has been given. (The main focus of Refs. $[10,11]$ is the study of memory effects.) For the case of scalar leptons, the vanishing of the asymmetry in equilibrium has been demonstrated recently in Refs. [12, 13].

In addition to offering the framework for a first-principle derivation of equations that describe leptogenesis, the CTP formalism also provides finite density corrections that do not straightforwardly follow in the Boltzmann approach. These corrections take the form of an integral over terms linear in the Higgs boson and lepton distribution functions. Historically, it has been proposed to compute the $C P$ asymmetry from the discontinuity of the correlation functions in the Matsubara (imaginary time) formalism. In the CTP (real time) approach, this corresponds to the imaginary part of the timeordered correlation functions $[14,15]$. It is characteristic of these Green functions, that they feature not only terms that are linear in the lepton and Higgs boson distribution functions, but also a product term of these distributions. In contrast, it appears to be the consensus among the more recent papers $[10,11,12,13]$ that the $C P$ asymmetry does not receive corrections from a product term of Higgs boson and lepton distribution functions. This has been shown in detail for the model with scalar leptons in Refs. [12, 13]. Here we find the same property in the model with fermionic leptons: The $C P$ asymmetry results from terms that can be written as the difference of Wightman type correlators (no explicit time-ordering of the field operators).

After deriving the kinetic equation for the lepton asymmetry in the CTP formalism we solve it numerically in various situations. We find that the effect of the new quantum statistical corrections is small when the bulk of the lepton asymmetry is produced at temperatures $T \ll M_{1}$, where $M_{1}$ is the mass of the neutrino that decays out of equilibrium, because the distribution functions are strongly Boltzmann suppressed for momenta much large than $T$. This situation corresponds to the strong-washout scenario, which is of particular interest since the resulting asymmetry is independent of both, the initial abundance of heavy neutrinos and a possible primordial lepton asymmetry. On the other hand, when the bulk of the asymmetry is produced for $T \sim M_{1}$ or larger, which corresponds to weak washout, the impact is more sizable. For a vanishing initial lepton asymmetry and thermal initial conditions of $N_{1}$, we find an enhancement of the asymmetry, as apparently the Bose enhancement from the Higgs particle distribution function dominates over the Fermi suppression from the lepton distribution. This qualitatively confirms what has been reported for scalar leptons [12, 13]. In this context, we note that the effects of finite density have been considered when the quantum statistical factors are accounted for in the initial and final states [16, 17], but not in the loop corrections, in which case it has also been found that the corrections may be sizable in the weak-washout regime. However, the quantum statistical factors in the loop corrections 
appear within the CTP approach on the same footing and with the same size as the external state factors, which is why they cannot be neglected consistently.

The remainder of this paper is organised as follows: In Section 2, we specify the model, introduce the free CTP propagators and provide the one-loop self energies. These ingredients are used in Section 3 to derive the Kadanoff-Baym equations that correspond to the tree-level Boltzmann equations with quantum statistical factors. The wave-function correction that leads to a $C P$ asymmetry arises from a two-loop diagram in the CTP formalism, which we discuss in Section 4. We explicitly show that no asymmetry is produced in equilibrium and we relate this fact to the usual procedure of RIS subtraction. Similarly, we discuss the vertex correction in Section 5. In Section 6, we numerically evaluate the effective $C P$-violating parameter and solve the Boltzmann equations for different strengths of washout, which demonstrates that finite density corrections are important for weak washout, but may be neglected in the strong-washout domain. We conclude in Section 7.

\section{CTP Approach to Leptogenesis}

In the simplest scenario of leptogenesis, there are two right handed neutrinos $N_{i}(i=1,2)$ with lepton-number violating Majorana masses $M_{i}$, one scalar $\mathrm{SU}(2)$ Higgs doublet $\phi$ and an active left-handed lepton doublet $\ell$. The Lagrangian of the model is

$$
\mathcal{L}=\frac{1}{2} \bar{\psi}_{N i}\left(\mathrm{i} \not \partial-M_{i}\right) \psi_{N i}+\bar{\psi}_{\ell} \mathrm{i} \not \partial \psi_{\ell}+\left(\partial^{\mu} \phi^{\dagger}\right)\left(\partial_{\mu} \phi\right)-Y_{i}^{*} \bar{\psi}_{\ell} \phi^{\dagger} P_{\mathrm{R}} \psi_{N i}-Y_{i} \bar{\psi}_{N i} P_{\mathrm{L}} \phi \psi_{\ell},
$$

where a summation over $i=1,2$ is understood and $P_{\mathrm{L} / \mathrm{R}}=\left(1 \mp \gamma_{5}\right) / 2$. Besides, it is implicitly assumed that the $\mathrm{SU}(2)$ indices are contracted in a gauge-invariant way, that is $\phi \psi_{\ell}=-\phi_{A} \varepsilon_{A B} \psi_{\ell B}$ and $\bar{\psi}_{\ell} \phi^{\dagger}=\left(\bar{\psi}_{\ell}\right)_{A} \varepsilon_{A B}\left(\phi^{\dagger}\right)_{B}$, where $\epsilon_{A B}$ denotes the antisymmetric $2 \times 2$ matrix with $\epsilon_{12}=1$. Upon the four-component spinor $\psi_{N i}$, we impose the Majorana constraint

$$
\psi_{N i}^{c}=C \bar{\psi}_{N i}^{T}=\psi_{N i}
$$

with $C$ the charge conjugation matrix.

\subsection{Propagators}

For the CTP formalism we follow closely the notational conventions of Refs. [18, 19, 20] and define the CTP propagators for the leptons by

$$
\begin{aligned}
& \mathrm{i} S_{\ell \alpha \beta}^{+-}(u, v)=\mathrm{i} S_{\ell \alpha \beta}^{<}(u, v)=-\left\langle\bar{\psi}_{\ell \beta}(v) \psi_{\ell \alpha}(u)\right\rangle, \\
& \mathrm{i} S_{\ell \alpha \beta}^{-+}(u, v)=\mathrm{i} S_{\ell \alpha \beta}^{>}(u, v)=\left\langle\psi_{\ell \alpha}(u) \bar{\psi}_{\ell \beta}(v)\right\rangle, \\
& \mathrm{i} S_{\ell \alpha \beta}^{++}(u, v)=\mathrm{i} S_{\ell \alpha \beta}^{T}(u, v)=\left\langle T\left(\psi_{\ell \alpha}(u) \bar{\psi}_{\ell \beta}(v)\right)\right\rangle, \\
& \mathrm{i} S_{\ell \alpha \beta}^{--}(u, v)=\mathrm{i} S_{\ell \alpha \beta}^{\bar{T}}(u, v)=\left\langle\bar{T}\left(\psi_{\ell \alpha}(u) \bar{\psi}_{\ell \beta}(v)\right)\right\rangle .
\end{aligned}
$$


We perform a perturbation and gradient expansion of the full Kadanoff-Baym equations that uses the tree-level propagators and vertices as elementary building blocks. The treelevel propagators are therefore given by the vacuum propagators plus a finite-density contribution proportional to the spectral function of the free theory. When there are no correlations between modes of different momentum and spin, we can characterize the state by particle number distribution functions. In case there is also no preferred direction for the spin, the usual averaging procedure applies. These assumptions lead to the explicit expressions

$$
\begin{aligned}
& \mathrm{i} S_{\ell}^{<}(p)=-2 \pi \delta\left(p^{2}\right) P_{\mathrm{L}} \not p P_{\mathrm{R}}\left[\vartheta\left(p_{0}\right) f_{\ell}(\mathbf{p})-\vartheta\left(-p_{0}\right)\left(1-\bar{f}_{\ell}(-\mathbf{p})\right)\right], \\
& \mathrm{i} S_{\ell}^{>}(p)=-2 \pi \delta\left(p^{2}\right) P_{\mathrm{L}} \not P_{\mathrm{R}}\left[-\vartheta\left(p_{0}\right)\left(1-f_{\ell}(\mathbf{p})\right)+\vartheta\left(-p_{0}\right) \bar{f}_{\ell}(-\mathbf{p})\right], \\
& \mathrm{i} S_{\ell}^{T}(p)=P_{\mathrm{L}} \frac{\mathrm{i} p}{p^{2}+\mathrm{i} \varepsilon} P_{\mathrm{R}}-2 \pi \delta\left(p^{2}\right) P_{\mathrm{L}} \not P_{\mathrm{R}}\left[\vartheta\left(p_{0}\right) f_{\ell}(\mathbf{p})+\vartheta\left(-p_{0}\right) \bar{f}_{\ell}(-\mathbf{p})\right], \\
& \mathrm{i} S_{\ell}^{\bar{T}}(p)=-P_{\mathrm{L}} \frac{\mathrm{i} \not p}{p^{2}-\mathrm{i} \varepsilon} P_{\mathrm{R}}-2 \pi \delta\left(p^{2}\right) P_{\mathrm{L}} \not p P_{\mathrm{R}}\left[\vartheta\left(p_{0}\right) f_{\ell}(\mathbf{p})+\vartheta\left(-p_{0}\right) \bar{f}_{\ell}(-\mathbf{p})\right],
\end{aligned}
$$

for the lepton propagators, where $f_{\ell}(\mathbf{k})$ denotes the distribution function of leptons and $\bar{f}_{\ell}(\mathbf{k})$ of anti-leptons. The leptons $\ell$ occur within an $\mathrm{SU}(2)$ doublet, but since this symmetry is unbroken at the time of leptogenesis, we assume that the lepton densities are $\mathrm{SU}(2)$-symmetric and hence diagonal. In particular, the lepton propagators defined here are the diagonal components of a diagonal propagator with additional SU(2) indices:

$$
S_{\ell A B}^{\mathrm{SU}(2)}(u, v)=\delta_{A B} S_{\ell}(u, v) \quad A, B=1,2 .
$$

Similarly, for the Majorana-fermion propagators we find

$$
\begin{aligned}
& \mathrm{i} S_{N i}^{<}(p)=-2 \pi \delta\left(p^{2}-M_{i}^{2}\right)\left(\not p+M_{i}\right)\left[\vartheta\left(p_{0}\right) f_{N i}(\mathbf{p})-\vartheta\left(-p_{0}\right)\left(1-f_{N i}(-\mathbf{p})\right)\right] \\
& \mathrm{i} S_{N i}^{>}(p)=-2 \pi \delta\left(p^{2}-M_{i}^{2}\right)\left(p+M_{i}\right)\left[-\vartheta\left(p_{0}\right)\left(1-f_{N i}(\mathbf{p})\right)+\vartheta\left(-p_{0}\right) f_{N i}(-\mathbf{p})\right] \\
& \mathrm{i} S_{N i}^{T}(p)=\frac{\mathrm{i}\left(\not p+M_{i}\right)}{p^{2}-M_{i}^{2}+\mathrm{i} \varepsilon}-2 \pi \delta\left(p^{2}-M_{i}^{2}\right)\left(\not p+M_{i}\right)\left[\vartheta\left(p_{0}\right) f_{N i}(\mathbf{p})+\vartheta\left(-p_{0}\right) f_{N i}(-\mathbf{p})\right] \\
& \mathrm{i} S_{N i}^{\bar{T}}(p)=-\frac{\mathrm{i}\left(\not p+M_{i}\right)}{p^{2}-M_{i}^{2}-\mathrm{i} \varepsilon}-2 \pi \delta\left(p^{2}-M_{i}^{2}\right)\left(\not p+M_{i}\right)\left[\vartheta\left(p_{0}\right) f_{N i}(\mathbf{p})+\vartheta\left(-p_{0}\right) f_{N i}(-\mathbf{p})\right]
\end{aligned}
$$

As a consequence of the Majorana condition (2), the distribution functions for neutrinos and anti-neutrinos are identical, and the propagators inherit the property

$$
S_{N i}(u, v)=C S_{N i}^{t}(v, u) C^{\dagger}
$$


where the transposition acts here on both the CTP and the Dirac indices. Finally the scalar propagators take the form

$$
\begin{aligned}
& \mathrm{i} \Delta_{\phi}^{<}(p)=2 \pi \delta\left(p^{2}\right)\left[\vartheta\left(p_{0}\right) f_{\phi}(\mathbf{p})+\vartheta\left(-p_{0}\right)\left(1+\bar{f}_{\phi}(-\mathbf{p})\right)\right], \\
& \mathrm{i} \Delta_{\phi}^{>}(p)=2 \pi \delta\left(p^{2}\right)\left[\vartheta\left(p_{0}\right)\left(1+f_{\phi}(\mathbf{p})\right)+\vartheta\left(-p_{0}\right) \bar{f}_{\phi}(-\mathbf{p})\right], \\
& \mathrm{i} \Delta_{\phi}^{T}(p)=\frac{\mathrm{i}}{p^{2}+\mathrm{i} \varepsilon}+2 \pi \delta\left(p^{2}\right)\left[\vartheta\left(p_{0}\right) f_{\phi}(\mathbf{p})+\vartheta\left(-p_{0}\right) \bar{f}_{\phi}(-\mathbf{p})\right], \\
& \mathrm{i} \Delta_{\phi}^{\bar{T}}(p)=-\frac{\mathrm{i}}{p^{2}-\mathrm{i} \varepsilon}+2 \pi \delta\left(p^{2}\right)\left[\vartheta\left(p_{0}\right) f_{\phi}(\mathbf{p})+\vartheta\left(-p_{0}\right) \bar{f}_{\phi}(-\mathbf{p})\right] .
\end{aligned}
$$

As for the leptons, it is understood that

$$
\Delta_{\phi A B}^{\mathrm{SU}(2)}(u, v)=\delta_{A B} \Delta_{\phi}(u, v) \quad A, B=1,2 .
$$

In the following, an occasional multiplicity factor $g_{w}=2$ arises from performing $\mathrm{SU}(2)$ traces such as

$$
\operatorname{tr}_{\mathrm{SU}(2)}\left[\Delta_{\phi}^{\mathrm{SU}(2)} \epsilon^{\dagger} S_{\ell}^{\mathrm{SU}(2)} \epsilon\right]=\Delta_{\phi} S_{\ell} \operatorname{tr}_{\mathrm{SU}(2)}\left[\epsilon^{\dagger} \epsilon\right]=g_{w} \Delta_{\phi} S_{\ell}
$$

In thermal equilibrium the distribution functions assume the usual Fermi-Dirac and Bose-Einstein forms, respectively:

$$
\begin{array}{r}
f_{\ell}(\mathbf{p})=\bar{f}_{\ell}(\mathbf{p})=f_{\ell}^{\mathrm{eq}}(\mathbf{p})=\frac{1}{e^{\beta|\mathbf{p}|}+1}, \\
f_{N i}(\mathbf{p})=f_{N i}^{\mathrm{eq}}(\mathbf{p})=\frac{1}{e^{\beta \sqrt{\mathbf{p}^{2}+M_{i}^{2}}}+1}, \\
f_{\phi}(\mathbf{p})=\bar{f}_{\phi}(\mathbf{p})=f_{\phi}^{\mathrm{eq}}(\mathbf{p})=\frac{1}{e^{\beta|\mathbf{p}|}-1} .
\end{array}
$$

Furthermore the equilibrium Green functions satisfy the Kubo-Martin-Schwinger (KMS) relation,

$$
\mathrm{i} S_{\ell}^{>}(p)=-\mathrm{e}^{\beta p_{0}} \mathrm{i} S_{\ell}^{<}(p), \quad \mathrm{i} S_{N i}^{>}(p)=-\mathrm{e}^{\beta p_{0}} \mathrm{i} S_{N i}^{<}(p), \quad \mathrm{i} \Delta_{\phi}^{>}(p)=\mathrm{e}^{\beta p_{0}} \mathrm{i} \Delta_{\phi}^{<}(p),
$$

an extremely useful property that we employ exhaustively throughout this paper.

\subsection{One-loop self-energies}

The one-loop neutrino self-energy in the CTP formalism is given by ( $c f$. the vacuum expression in Ref. [21])

$$
\begin{aligned}
\mathrm{i} Z_{N i j}^{a b}(k)= & g_{w} \int \frac{d^{4} k^{\prime}}{(2 \pi)^{4}} \frac{d^{4} k^{\prime \prime}}{(2 \pi)^{4}}(2 \pi)^{4} \delta^{(4)}\left(k-k^{\prime}-k^{\prime \prime}\right) \\
& \times\left\{Y_{i} Y_{j}^{*} P_{\mathrm{L}} \mathrm{i} S_{\ell}^{a b}\left(k^{\prime}\right) P_{\mathrm{R}} \mathrm{i} \Delta_{\phi}^{a b}\left(k^{\prime \prime}\right)+Y_{i}^{*} Y_{j} C\left[P_{\mathrm{L}} \mathrm{i} S_{\ell}^{b a}\left(-k^{\prime}\right) P_{\mathrm{R}}\right]^{T} C^{\dagger} \mathrm{i} \Delta_{\phi}^{b a}\left(-k^{\prime \prime}\right)\right\},
\end{aligned}
$$


where $a, b= \pm$ denote the CTP indices, see Eqs. (3a)-(3d). Our definition for the selfenergies is such that $-i \Sigma$ corresponds to the sum of all one-particle irreducible diagrams. The one-loop lepton self-energy reads

$$
\mathrm{i}_{\ell}^{a b}(k)=\left|Y_{i}\right|^{2} \int \frac{d^{4} k^{\prime}}{(2 \pi)^{4}} \frac{d^{4} k^{\prime \prime}}{(2 \pi)^{4}}(2 \pi)^{4} \delta^{(4)}\left(k-k^{\prime}-k^{\prime \prime}\right) P_{\mathrm{R}} \mathrm{i} S_{N i}^{a b}\left(k^{\prime}\right) P_{\mathrm{L}} \mathrm{i} \Delta_{\phi}^{b a}\left(-k^{\prime \prime}\right),
$$

where we sum over the neutrino flavours $i$. In thermal equilibrium these self-energies inherit the KMS property

$$
\mathcal{Y}_{N i, \ell}^{>}(p)=-\mathrm{e}^{\beta p_{0}} \Psi_{N i, \ell}^{<}(p)
$$

from the constituent CTP Green functions. At the one-loop order it is easy to verify this explicitly by inserting the tree-level propagators (4), (6), (8) into the self-energies (13), (14). One of the main objectives of this paper is to show explicitly how KMS also remains valid when considering the next-to-leading order contributions to $\mathbb{Z}_{\mathrm{Ni, \ell},}^{<,>}$. It turns out that this encompasses the subtraction of RIS as it is performed in the conventional approach to baryo- and leptogenesis.

\section{Tree-Level Contributions to the Kinetic Equations}

The theoretical description of leptogenesis is concerned with the time evolution of nonequilibrium densities of active leptons and of right-handed neutrinos. This is described by the Kadanoff-Baym equations (see e.g. $[18,19,20]$ ). We perform the standard Wigner transformation and gradient expansion. Since we are interested in finite-density effects from loops in the CTP formalism, we restrict ourselves to the leading term in the gradient expansion. We regard this as a self-consistent leading-order approximation to the full Kadanoff-Baym equations, relative to which corrections from higher gradients and loops may eventually be considered. Thus, the kinetic equations take the form

$$
\begin{aligned}
& \frac{d}{d t} \gamma_{0} \mathrm{i} S_{\ell}^{<,>}(k)=-\left[\mathrm{i} Z_{\ell}^{>}(k) P_{\mathrm{L}} \mathrm{i} S_{\ell}^{<}(k)-\mathrm{i} Z_{\ell}^{<}(k) P_{\mathrm{L}} \mathrm{i} S_{\ell}^{>}(k)\right], \\
& \frac{d}{d t} \gamma_{0} \mathrm{i} S_{N i}^{<,>}(k)=-\left[\mathrm{i} \not_{N i i}^{>}(k) \mathrm{i} S_{N i}^{<}(k)-\mathrm{i} Z_{N i i}^{<}(k) \mathrm{i} S_{N i}^{>}(k)\right],
\end{aligned}
$$

where we recognize the familiar expression for the collision term in the CTP formalism on the right-hand side. Here and in the following we assume spatial isotropy, which means that there is no angular dependence in the distribution functions and we may identify $f_{\ell, \phi, N i}(\mathbf{k}) \equiv f_{\ell, \phi, N i}(|\mathbf{k}|)$. In particular, we repeatedly use $f_{\ell, \phi, N i}(-\mathbf{k})=f_{\ell, \phi, N i}(\mathbf{k})$ to simplify the arguments of distribution functions. When performing the Wigner transformation and gradient expansion in a slowly varying background, all time dependence in the propagators $(4),(6),(8)$ is isolated in the distribution functions $f_{\ell, \phi, N i}$, where we suppress an explicit time argument. The equation for the lepton density can be simplified when taking the Dirac trace of Eq. (16a), multiplying by minus one and integrating 
over $k_{0}$. Inserting Eq. (4), we obtain

$$
\frac{d}{d t}\left(f_{\ell}(\mathbf{k})-\bar{f}_{\ell}(\mathbf{k})\right)=\mathcal{C}_{\ell}(\mathbf{k})=\int \frac{d k_{0}}{2 \pi} \operatorname{tr}\left[\mathrm{i} \not_{\ell}^{>}(k) P_{\mathrm{L}} \mathrm{i} S_{\ell}^{<}(k)-\mathrm{i} \not_{\ell}^{<}(k) P_{\mathrm{L}} \mathrm{i} S_{\ell}^{>}(k)\right],
$$

which defines $\mathcal{C}_{\ell}(\mathbf{k})$. In a similar way we obtain the equation for the time dependence of the Majorana neutrino distribution function

$$
\frac{d}{d t} f_{N i}(\mathbf{k})=\mathcal{C}_{N i}(\mathbf{k})=\frac{1}{4} \int \frac{d k_{0}}{2 \pi} \operatorname{sign}\left(k_{0}\right) \operatorname{tr}\left[\mathrm{i} \mathbb{Z}_{N i i}^{>}(k) \mathrm{i} S_{N i}^{<}(k)-\mathrm{i} \mathbb{Z}_{N i i}^{<}(k) \mathrm{i} S_{N i}^{>}(k)\right] .
$$

\subsection{Tree-level collision terms}

The tree-level collision term for the leptons can be straightforwardly evaluated by inserting the tree-level propagators into Eq. (17) and the expression (14) for the self-energy. Since we are interested only in the lepton number density

$$
n_{l}=\int \frac{d^{3} k}{(2 \pi)^{3}} f_{l}(\mathbf{k})
$$

we integrate $\mathcal{C}_{\ell}(\mathbf{k})$ over $\mathbf{k}$ and find

$$
\begin{aligned}
\int \frac{d^{3} k}{(2 \pi)^{3}} & \mathcal{C}_{\ell}(\mathbf{k})=-\left|Y_{i}\right|^{2} \int \frac{d^{3} k}{(2 \pi)^{3} 2\left|\mathbf{k}^{2}\right|} \frac{d^{3} k^{\prime}}{(2 \pi)^{3} 2 \sqrt{\mathbf{k}^{\prime 2}+M_{i}^{2}}} \frac{d^{3} k^{\prime \prime}}{(2 \pi)^{3} 2\left|\mathbf{k}^{\prime \prime}\right|} \\
\times & (2 \pi)^{4} \delta^{4}\left(k^{\prime}-k-k^{\prime \prime}\right) 2 k^{\prime} \cdot k\left\{\left(1-f_{N i}\left(\mathbf{k}^{\prime}\right)\right) \times\left[f_{\ell}(\mathbf{k}) f_{\phi}\left(\mathbf{k}^{\prime \prime}\right)-\bar{f}_{\ell}(\mathbf{k}) \bar{f}_{\phi}\left(\mathbf{k}^{\prime \prime}\right)\right]\right. \\
& \left.-f_{N i}\left(\mathbf{k}^{\prime}\right) \times\left[\left(1-f_{\ell}(\mathbf{k})\right)\left(1+f_{\phi}\left(\mathbf{k}^{\prime \prime}\right)\right)-\left(1-\bar{f}_{\ell}(\mathbf{k})\right)\left(1+\bar{f}_{\phi}\left(\mathbf{k}^{\prime \prime}\right)\right)\right]\right\} .
\end{aligned}
$$

Due to the fast violation of Higgs number, we can assume that $f_{\phi}=\bar{f}_{\phi} \equiv f_{\phi}^{\text {eq }}$. For the leptons we may assume $f_{\ell}-f_{\ell}^{\text {eq }}=-\left(\bar{f}_{\ell}-f_{\ell}^{\text {eq }}\right)$, as justified by fast pair-annihilating and -creating interactions among the leptons. Expanding to linear order in the deviations from equilibrium, the lepton collision term simplifies to

$$
\begin{aligned}
\int \frac{d^{3} k}{(2 \pi)^{3}} \mathcal{C}_{\ell}(\mathbf{k})= & -\left|Y_{i}\right|^{2} \int \frac{d^{3} k}{(2 \pi)^{3} 2|\mathbf{k}|} \frac{d^{3} k^{\prime}}{(2 \pi)^{3} 2 \sqrt{\mathbf{k}^{\prime 2}+M_{i}^{2}}} \frac{d^{3} k^{\prime \prime}}{(2 \pi)^{3} 2\left|\mathbf{k}^{\prime \prime}\right|} \\
& \times(2 \pi)^{4} \delta^{4}\left(k^{\prime}-k-k^{\prime \prime}\right) 2 k^{\prime} \cdot k\left[f_{\phi}^{\mathrm{eq}}\left(\mathbf{k}^{\prime \prime}\right)+f_{N i}\left(\mathbf{k}^{\prime}\right)\right] \times\left[f_{\ell}(\mathbf{k})-\bar{f}_{\ell}(\mathbf{k})\right] .
\end{aligned}
$$

This term describes the washout of a lepton asymmetry through inverse decays. In a similar way we obtain the tree-level collision terms for the neutrinos by inserting the tree-level propagators into Eq. (18) and the expression (14) for the self-energy. For the neutrinos, we shall need the phase-space distributions, so we need the unintegrated 
collision term, for which we find

$$
\begin{aligned}
& \mathcal{C}_{N i}(\mathbf{k})=\frac{1}{4} g_{w}\left|Y_{i}\right|^{2} \frac{1}{2 \sqrt{\mathbf{k}^{2}+M_{i}^{2}}} \int \frac{d^{3} k^{\prime}}{(2 \pi)^{3} 2\left|\mathbf{k}^{\prime}\right|} \frac{d^{3} k^{\prime \prime}}{(2 \pi)^{3} 2\left|\mathbf{k}^{\prime \prime}\right|}(2 \pi)^{4} \delta^{4}\left(k-k^{\prime}-k^{\prime \prime}\right) \\
& \times \operatorname{tr}\left[\not k^{\prime}\left(\not k+M_{i}\right)\left\{-f_{N i}(\mathbf{k})\left[1-f_{\ell}\left(\mathbf{k}^{\prime}\right)\right]\left[1+f_{\phi}\left(\mathbf{k}^{\prime \prime}\right)\right]+\left[1-f_{N i}(\mathbf{k})\right] f_{\ell}\left(\mathbf{k}^{\prime}\right) f_{\phi}\left(\mathbf{k}^{\prime \prime}\right)\right\}\right. \\
&\left.+\not k^{\prime}\left(\not k+M_{i}\right)\left\{-f_{N i}(\mathbf{k})\left[1-\bar{f}_{\ell}\left(\mathbf{k}^{\prime}\right)\right]\left[1+\bar{f}_{\phi}\left(\mathbf{k}^{\prime \prime}\right)\right]+\left[1-f_{N i}(\mathbf{k})\right] \bar{f}_{\ell}\left(\mathbf{k}^{\prime}\right) \bar{f}_{\phi}\left(\mathbf{k}^{\prime \prime}\right)\right\}\right] .
\end{aligned}
$$

It is useful to check that when setting the Higgs and lepton distribution functions to zero, this reproduces the negative total decay rate of $N_{i}$,

$$
\mathcal{C}_{N i}^{f, \bar{f}_{\ell, \phi}=0}(\mathbf{k})=-\frac{g_{w}}{16 \pi}\left|Y_{i}^{2}\right| \frac{M_{i}^{2}}{\sqrt{\mathbf{k}^{2}+M_{i}^{2}}} f_{N i}(\mathbf{k}) .
$$

Next, introducing

$$
\delta f_{N i}=f_{N i}-f_{N i}^{\mathrm{eq}}
$$

and expanding to linear order around equilibrium distributions the Majorana collision term simplifies to

$$
\begin{aligned}
\mathcal{C}_{N i}(\mathbf{k})= & -g_{w}\left|Y_{i}\right|^{2} \frac{1}{2 \sqrt{\mathbf{k}^{2}+M_{i}^{2}}} \int \frac{d^{3} k^{\prime}}{(2 \pi)^{3} 2\left|\mathbf{k}^{\prime}\right|} \frac{d^{3} k^{\prime \prime}}{(2 \pi)^{3} 2\left|\mathbf{k}^{\prime \prime}\right|}(2 \pi)^{4} \delta^{4}\left(k-k^{\prime}-k^{\prime \prime}\right) \\
& \times 2 k \cdot k^{\prime} \delta f_{N i}(\mathbf{k})\left[1-f_{\ell}^{\mathrm{eq}}\left(\mathbf{k}^{\prime}\right)+f_{\phi}^{\mathrm{eq}}\left(\mathbf{k}^{\prime \prime}\right)\right] .
\end{aligned}
$$

Note that $\delta f_{N i}$ is not necessarily small in some realistic scenarios for leptogenesis, e.g. for weak washout.

It is easy to see from Eqs (21), (25) that in thermal equilibrium the collision terms vanish. Alternatively, one may either by directly use the KMS relations $(12,15)$ in Eqs. $(17,18)$ or explicitly insert the equilibrium distributions into Eqs. $(20,22)$. This means that the equilibrium state is a static (time-independent) solution to the KadanoffBaym equations.

\subsection{Expansion of the Universe}

Before proceeding we comment on how the effects of particle dilution due to the expansion of the Universe can be systematically incorporated into our equations. So far we have formulated the Kadanoff-Baym equation on the Minkowski background. Neglecting Planck-scale suppressed corrections the only effect of replacing the Minkowski background by the Friedmann-Robertson-Walker one is that the particle modes are redshifted.

This can be conveniently implemented by adopting conformal coordinates, which are defined by the metric

$$
g_{\mu \nu}=a^{2}(\eta) \operatorname{diag}(1,-1,-1,-1)
$$


where $\eta=x^{0}$ denotes the conformal time and $a(\eta)$ the scale factor. Then the Lagrangian (1) describes the dynamics in the expanding background, provided that we have redefined the fields such that their kinetic terms are canonically normalized, that we replace $M_{i} \rightarrow a M_{i}$ and that we assume that the Higgs field has no coupling to the scalar curvature $R$. Consequently all expressions given earlier in this Section and in Sections 4 and 5 below remain valid in the expanding Universe if momenta $\mathbf{k}$ are understood as comoving momenta $\mathbf{k}_{\mathrm{com}}$, temperature $T$ (and $\beta=1 / T$ ) as comoving temperature $T_{\text {com }}$ (and $\beta_{\text {com }}$ ), time $t$ as conformal time $\eta, f\left(\mathbf{k}_{\text {com }}\right)$ and $n$ as comoving phase-space distributions and particle number densities, respectively, and if finally masses $M_{i}$ are replaced by $a M_{i}$, wherever they appear.

For the scale factor in the radiation-dominated Universe we use the relation

$$
a(\eta)=a_{\mathrm{R}} \eta
$$

Using the Friedmann equation and the free energy, we can relate this to the temperature as

$$
T=\frac{T_{\mathrm{com}}}{a(\eta)}=\frac{1}{a(\eta)} \sqrt{\frac{a_{\mathrm{R}} m_{\mathrm{Pl}}}{2}}\left(\frac{45}{g_{*} \pi^{3}}\right)^{1 / 4},
$$

where $g_{*}$ denotes the number of relativistic degrees of freedom and $m_{\mathrm{Pl}}=1 / \sqrt{G_{N}}=$ $1.22 \cdot 10^{19} \mathrm{GeV}$ the Planck mass. The variable $z=M_{1} / T$ that will be used in Section 6 is therefore related to $\eta$ by a constant proportionality factor.

Kinetic equations are often formulated in terms of physical momenta and physical time. In order to make contact, we can replace in our equations the comoving momenta by physical momenta $k_{\mathrm{ph}}=k_{\mathrm{com}} / a$ and the comoving temperature by the physical temperature $T_{\mathrm{ph}}=T_{\mathrm{com}} / a$. Doing so and dividing by a factor of $a$ for convenience, the right-hand sides (i.e. the collision terms), simply take the same form as given earlier in this Section, where now all momenta are to be understood as physical. The left-hand sides change according to

$$
\begin{aligned}
\frac{1}{a(\eta)} \frac{d}{d \eta} f\left(\mathbf{k}_{\mathrm{com}}\right) & =\frac{\partial}{\partial t} f\left(\mathbf{k}_{\mathrm{ph}}\right)+\left(\frac{\partial}{\partial\left|\mathbf{k}_{\mathrm{ph}}\right|} f\left(\mathbf{k}_{\mathrm{ph}}\right)\right) \frac{\partial\left|\mathbf{k}_{\mathrm{ph}}\right|}{\partial t} \\
& =\frac{\partial}{\partial t} f\left(\mathbf{k}_{\mathrm{ph}}\right)-H\left|\mathbf{k}_{\mathrm{ph}}\right| \frac{\partial}{\partial\left|\mathbf{k}_{\mathrm{ph}}\right|} f\left(\mathbf{k}_{\mathrm{ph}}\right),
\end{aligned}
$$

where $f$ may stand for $f_{N}$ or $f_{\ell}-\bar{f}_{\ell}$. This formula applies to the treatment of the densities of right-handed neutrinos, that do not necessarily maintain kinetic equilibrium during leptogenesis, implying that one cannot substitute for $f_{N i}$ a Fermi-Dirac distribution with a (pseudo-)chemical potential. For the lepton charge, it is suitable to consider the integrated version

$$
\frac{1}{a(\eta)} \int \frac{d^{3} k_{\mathrm{ph}}}{(2 \pi)^{3}} \frac{d}{d \eta} f\left(\mathbf{k}_{\mathrm{com}}\right)=\frac{\partial}{\partial t} n_{\mathrm{ph}}+3 H n_{\mathrm{ph}}
$$

of the above equation. This is just the familiar cosmological dilution law, where $n_{\mathrm{ph}}$ denotes a physical number density and $H$ the Hubble parameter with respect to time $t$. 
However, in Section 6 we will solve the kinetic equations directly in conformal coordinates.

\subsection{Boltzmann equations}

We now write down explicitly the Boltzmann equations that describe the time evolution of the distribution function $f_{N 1}$ and of the lepton asymmetry in conformal coordinates:

$$
\begin{aligned}
\frac{d}{d \eta} f_{N 1}\left(\mathbf{k}_{\mathrm{com}}\right) & =D\left(\mathbf{k}_{\mathrm{com}}\right), \\
\frac{d}{d \eta}\left(n_{\ell}-\bar{n}_{\ell}\right) & =W+S .
\end{aligned}
$$

Here $D\left(\mathbf{k}_{\text {com }}\right)$ denotes the contributions (25) from decays and inverse decays of $N_{1}$, while $W$ denotes the washout term (21). $S=S^{\mathrm{wf}}+S^{\mathrm{v}}$ is the source term for the lepton asymmetry, which we decompose into a wave-function contribution $S^{\text {wf }}$ and a vertex contribution $S^{\mathrm{v}}$. Note that here and below, unless indicated otherwise, $n_{\ell}$ refers to the comoving particle number density.

Performing the substitution of variables pertinent to conformal coordinates as discussed above in Eq. (22), we obtain:

$$
\begin{aligned}
D\left(\mathbf{k}_{\mathrm{com}}\right)= & 2 g_{w}\left|Y_{1}\right|^{2} \frac{1}{2 \sqrt{\mathbf{k}_{\mathrm{com}}^{2}+a^{2}(\eta) M_{1}^{2}}} \int \frac{d^{3} k_{\mathrm{com}}^{\prime}}{(2 \pi)^{3} 2\left|\mathbf{k}_{\mathrm{com}}^{\prime}\right|} \frac{d^{3} k_{\mathrm{com}}^{\prime \prime}}{(2 \pi)^{3} 2\left|\mathbf{k}_{\mathrm{com}}^{\prime \prime}\right|} \\
& \times(2 \pi)^{4} \delta^{4}\left(k_{\mathrm{com}}-k_{\mathrm{com}}^{\prime}-k_{\mathrm{com}}^{\prime \prime}\right) k_{\mathrm{com}} \cdot k_{\mathrm{com}}^{\prime} \\
& \times\left[-f_{N 1}\left(\mathbf{k}_{\mathrm{com}}\right)\left(1-f_{\ell}\left(\mathbf{k}_{\mathrm{com}}\right)+f_{\phi}\left(\mathbf{k}_{\mathrm{com}}^{\prime \prime}\right)\right)+f_{\ell}\left(\mathbf{k}_{\mathrm{com}}^{\prime}\right) f_{\phi}\left(\mathbf{k}_{\mathrm{com}}^{\prime \prime}\right)\right] .
\end{aligned}
$$

In the evaluation of the tree-level collision term we may set $f_{\ell}$ and $f_{\phi}$ equal to the equilibrium distributions given in Eq. (11) (but expressed in terms of comoving momentum and temperature), which allows us to evaluate the phase-space integrals. It proves useful to define the quantity

$$
\Sigma_{N}^{\mu}(k)=g_{w} \int \frac{d^{3} p}{(2 \pi)^{3} 2|\mathbf{p}|} \frac{d^{3} q}{(2 \pi)^{3} 2|\mathbf{q}|}(2 \pi)^{4} \delta^{4}(k-p-q) p^{\mu}\left(1-f_{\ell}^{\mathrm{eq}}(\mathbf{p})+f_{\phi}^{\mathrm{eq}}(\mathbf{q})\right),
$$

which we will calculate analytically in Section 6. In terms of this we find for the decay and inverse decay term

$$
D\left(\mathbf{k}_{\mathrm{com}}\right)=-2\left|Y_{1}\right|^{2} \frac{k_{\mathrm{com} \mu}}{2 k_{\mathrm{com} 0}} \Sigma_{N}^{\mu}\left(k_{\mathrm{com}}\right)\left[f_{N 1}\left(\mathbf{k}_{\mathrm{com}}\right)-f_{N 1}^{\mathrm{eq}}\left(\mathbf{k}_{\mathrm{com}}\right)\right],
$$

where $k_{\mathrm{com} 0}=\sqrt{\mathbf{k}_{\mathrm{com}}^{2}+a_{\mathrm{R}}^{2} \eta^{2} M_{1}^{2}}$ and

$$
f_{N 1}^{\mathrm{eq}}\left(\mathbf{k}_{\mathrm{com}}\right)=\frac{1}{\mathrm{e}^{k_{\mathrm{com} 0} / T_{\mathrm{com}}}+1} .
$$


To calculate the washout term (21) we approximate $f_{N i}$ by the equilibrium distribution $f_{N i}^{\text {eq }}$ and assume that the leptons are in kinetic equilibrium so that $f_{\ell}$ can be parameterized in terms of a chemical potential. Expanding to linear order in the chemical potential, the latter assumption yields the relation

$$
f_{\ell}(\mathbf{k})-\bar{f}_{\ell}(\mathbf{k})=\left(n_{l}-\bar{n}_{l}\right) \times \frac{12 \beta^{3} e^{\beta|\mathbf{k}|}}{\left(e^{\beta|\mathbf{k}|}+1\right)^{2}} .
$$

We then obtain for the wash-out term

$$
W=\int \frac{d^{3} k}{(2 \pi)^{3}} \mathcal{C}_{\ell}(\mathbf{k})=-\frac{3\left|Y_{1}\right|^{2}}{8 \pi^{3}} \frac{a^{2} M_{1}^{2}}{T_{\mathrm{com}}}\left(n_{\ell}-\bar{n}_{\ell}\right) \int_{0}^{\infty} d x \frac{\mathrm{e}^{x}}{\left(\mathrm{e}^{x}+1\right)^{2}} \ln \left(\frac{\mathrm{e}^{\frac{a^{2} M_{1}^{2}}{4 T_{\mathrm{com}}^{2}}+x}+1}{\mathrm{e}^{\frac{a^{2} M_{1}^{2}}{4 T_{\mathrm{com}}^{2} x}+x}-\mathrm{e}^{x}}\right) .
$$

The substitution $f_{N i} \rightarrow f_{N i}^{\text {eq }}$, which was made to take analytical integrations further, is strictly speaking only a correct approximation when $\delta f_{N i} \ll f_{N i}^{\mathrm{eq}}$. As in scenarios of strong washout or in the transition region from weak to strong washout most of the asymmetry is eventually produced when $N_{1}$ is close to equilibrium, the error from assuming an equilibrium abundance of $N_{1}$ in the washout term at all times should be small in the final result. (In fact, when assuming Maxwell statistics $[2,15,22]$ the absence of Pauli blocking factors in Eq. (20) implies an even more drastic approximation, since then $f_{N i}\left(\mathbf{k}^{\prime}\right) \rightarrow 0$ in Eq. (21).)

As is well known, the source term $S$ for the lepton asymmetry in Eq. (31) only arises in fourth order in the couplings $Y_{i}$. Deriving this source term including all quantum statistical factors is the subject of the following two sections.

\section{Self-Energy Contribution to the $C P$ Asymmetry}

A key ingredient to baryogenesis calculations in the conventional framework is the subtraction of RIS. If this were omitted, a baryon asymmetry would result in thermal equilibrium in contradiction with the CPT theorem and the unitarity of scattering matrices. In this Section we show how the RIS subtraction arises within the CTP framework for leptogenesis and derive the $C P$ asymmetry from the wave-function correction in the presence of finite number densities.

\subsection{Wave-Function Correction to $\mathcal{C}_{\ell}$}

The aim is the calculation of the wave-function correction to the lepton collision term (17),

$$
\mathcal{C}_{\ell}^{\mathrm{wf}}(\mathbf{k})=\int \frac{d k_{0}}{2 \pi} \operatorname{tr}\left[\mathrm{i} \not_{\ell}^{\mathrm{wf}>}(k) P_{\mathrm{L}} \mathrm{i} S_{\ell}^{<}(k)-\mathrm{i} \gtrless_{\ell}^{\mathrm{wf}<}(k) P_{\mathrm{L}} \mathrm{i} S_{\ell}^{>}(k)\right]
$$


where the correction to the lepton self-energy is

$$
\mathrm{i} \ddot{Y}_{\ell}^{\mathrm{wf}<,>}(k)=\int \frac{d^{4} k^{\prime}}{(2 \pi)^{4}} \frac{d^{4} k^{\prime \prime}}{(2 \pi)^{4}}(2 \pi)^{4} \delta^{4}\left(k-k^{\prime}-k^{\prime \prime}\right) Y_{i}^{*} Y_{j} P_{\mathrm{R}} \mathrm{i} S_{N i j}^{\mathrm{wf}<,>}\left(k^{\prime}\right) P_{\mathrm{L}} \mathrm{i} \Delta_{\phi}^{>,<}\left(-k^{\prime \prime}\right) \text {. }
$$

In contrast to Eq. (20) the propagator $\mathrm{i} S_{N i j}^{\mathrm{wf}<,>}$ in this expression is not the tree propagator, but the propagator including a one-loop self-energy correction. Hence, we must also allow for off-diagonal terms and obtain for the wave-function corrections to the neutrino Wightman functions:

$$
\begin{aligned}
& -\mathrm{i} S_{N i j}^{\mathrm{wf}>}\left(k^{\prime}\right)=\mathrm{i} S_{N i}^{>} \mathrm{i} Z_{N i j}^{T} \mathrm{i} S_{N j}^{T}-\mathrm{i} S_{N i}^{\bar{T}} \mathrm{i} \Psi_{N i j}^{>} \mathrm{i} S_{N j}^{T}-\mathrm{i} S_{N i}^{>} \mathrm{i} \Psi_{N i j}^{<} \mathrm{i} S_{N j}^{>}+\left.\mathrm{i} S_{N i}^{\bar{T}} \mathrm{i} Z_{N i j}^{\bar{T}} \mathrm{i} S_{N j}^{>}\right|_{k^{\prime}}, \\
& -\mathrm{i} S_{N i j}^{\mathrm{wf}<}\left(k^{\prime}\right)=\mathrm{i} S_{N i}^{<} \mathrm{i} Z_{N i j}^{\bar{T}} \mathrm{i} S_{N j}^{\bar{T}}-\mathrm{i} S_{N i}^{T} \mathrm{i} \not_{N i j}^{<}{ }_{i j} \mathrm{i} S_{N j}^{\bar{T}}-\mathrm{i} S_{N i}^{<} \mathrm{i} Z_{N i j}^{>} \mathrm{i} S_{N j}^{<}+\left.\mathrm{i} S_{N i}^{T} \mathrm{i} Z_{N i j}^{T} \mathrm{i} S_{N j}^{<}\right|_{k^{\prime}},
\end{aligned}
$$

The different signs are a consequence of the Feynman rules on the CTP, and all functions on the right-hand side carry momentum argument $k^{\prime}$ as indicated after the bar.

In order to reduce the number of terms that we need to write, we assume that $M_{2} \gg M_{1}$, such that the density of $N_{2}$ can be neglected at the time of leptogenesis. In that case, the off-diagonal components reduce to

$$
\begin{aligned}
& -\mathrm{i} S_{N 12}^{\mathrm{wf}>}=\mathrm{i} S_{N 1}^{>} \mathrm{i} \Psi_{N 12}^{T} \mathrm{i} S_{N 2}^{T}-\mathrm{i} S_{N 1}^{\bar{T}} \mathrm{i} \Psi_{N 12}^{>} \mathrm{i} S_{N 2}^{T}, \\
& -\mathrm{i} S_{N 12}^{\mathrm{wf}<}=\mathrm{i} S_{N 1}^{<} \mathrm{i} \Psi_{N 12}^{\bar{T}} \mathrm{i} S_{N 2}^{\bar{T}}-\mathrm{i} S_{N 1}^{T} \mathrm{i} \psi_{N 12}^{<} \mathrm{i} S_{N 2}^{\bar{T}}, \\
& -\mathrm{i} S_{N 21}^{\mathrm{wf}>}=-\mathrm{i} S_{N 2}^{\bar{T}} \mathrm{i} ⿱_{N 21}^{>} \mathrm{i} S_{N 1}^{T}+\mathrm{i} S_{N 2}^{\bar{T}} \mathrm{i} Z_{N 21}^{\bar{T}} \mathrm{i} S_{N 1}^{>} \text {, } \\
& -\mathrm{i} S_{N 21}^{\mathrm{wf}<}=-\mathrm{i} S_{N 2}^{T} \mathrm{i} \Psi_{N 21}^{<} \mathrm{i} S_{N 1}^{\bar{T}}+\mathrm{i} S_{N 2}^{T} \mathrm{i} \Psi_{N 21}^{T} \mathrm{i} S_{N 1}^{<} .
\end{aligned}
$$

As long as $\left|M_{2}-M_{1}\right| \gg \Gamma_{N 1}, \Gamma_{N 2}$, where $\Gamma_{N i}$ are the total decay rates of $N_{i}$, we can straightforwardly extend the present analysis to situations where $M_{2} \gg M_{1}$ does not hold by simply adding the corresponding contributions that arise from a non-vanishing density of $N_{2}$, i.e. the asymmetry produced in $N_{2}$ decays. The reason for this is that not both of the neutrinos in the wave-function diagram can be on-shell simultaneously, provided their mass terms are not degenerate. Because of this we do not further simplify $\mathrm{i} S_{N 2}^{T, \bar{T}}$ to $-\mathrm{i} / M_{2}$ at this point.

\subsection{KMS and Real Intermediate State Subtraction}

It follows from Eq. (38) that $\mathcal{C}_{\ell}^{\text {wf }}$ vanishes provided both $\mathbb{\psi}_{\ell}^{\mathrm{wf}<,>}$ and $S_{\ell}^{<,>}$satisfy the KMS relation. The correction $\mathcal{Z}_{\ell}^{\mathrm{wf}<,>}$ in turn satisfies KMS provided it also holds for $\mathrm{i} S_{N i j}^{\mathrm{wf}<,>}$, see Eq. (39). It is instructive to show this property,

$$
\mathrm{i} S_{N i j}^{\mathrm{wf}>}(k)=-\mathrm{e}^{\beta k_{0}} \mathrm{i} S_{N i j}^{\mathrm{wf}<}(k)
$$


explicitly, because it illustrates how the RIS subtraction is realised within the CTP framework.

To show the validity of the KMS relation for the wave-function correction (42) explicitly, we define

$$
\mathcal{K}_{i j}=\mathrm{i} S_{N i j}^{\mathrm{wf}>}(k)+e^{\beta k_{0}} \mathrm{i} S_{N i j}^{\mathrm{wf}<}(k) .
$$

The claim is $\mathcal{K}_{i j}=0$ when substituting equilibrium distributions. We show this on the example $\mathcal{K}_{12}$, from which it will be clear how to generalize to all components of $\mathcal{K}_{i j}$. Applying KMS to $\mathbb{Z}_{N 12}^{<,>}$and $S_{N 1}^{<,>}$, we find

$$
\mathcal{K}_{12}=-\mathrm{i} S_{N 1}^{>} \mathrm{i}\left(\bigotimes_{N 12}^{T}+\Psi_{N 12}^{\bar{T}}\right) \mathrm{i} S_{N 2}^{T}+\mathrm{i} S_{N 1}^{T} \mathrm{i} \Psi_{N 12}^{>} \mathrm{i} S_{N 2}^{T}+\mathrm{i} S_{N 1}^{\bar{T}} \mathrm{i} \Psi_{N 12}^{>} \mathrm{i} S_{N 2}^{T} .
$$

In addition, we have used that $S_{N 2}$ is evaluated here only when $N_{2}$ is off-shell, such that we can set $S_{N 2}^{\bar{T}}=-S_{N 2}^{T}$. To further simplify this expression, we make use of the following identities:

$$
\begin{gathered}
S_{N \mathrm{i}}^{T}+S_{N \mathrm{i}}^{\bar{T}}=S_{N \mathrm{i}}^{>}+S_{N \mathrm{i}}^{<}, \\
\psi_{N i j}^{T}+\Downarrow_{N i j}^{\bar{T}}=\gtrless_{N i j}^{>}+\gtrless_{N i j}^{<} .
\end{gathered}
$$

Note that the first identity, applied to the heavy neutrino $N_{2}$, recovers the property $S_{N 2}^{T}=-S_{N 2}^{\bar{T}}$, when evaluated for off-shell momenta. It follows that

$$
\mathcal{K}_{12}=-\mathrm{i} S_{N 1}^{>} \mathrm{i}\left(\Psi_{N 12}^{<}+\gtrless_{N 12}^{>}\right) \mathrm{i} S_{N 2}^{T}+\mathrm{i}\left(S_{N 1}^{<}+\mathrm{i} S_{N 1}^{>}\right) \mathrm{i} Z_{N 12}^{>} \mathrm{i} S_{N 2}^{T}=0,
$$

where we have once more applied the KMS relation to obtain the last equality. An immediate consequence of this result is that no asymmetry is produced in thermal equilibrium, as required.

Besides verifying the KMS relation for $S_{N i j}^{<,>}$and consequently also for $\Psi_{N i j}^{<,>}$and $\Psi_{\ell}^{<,>}$, the above calculation shows how the RIS subtraction emerges in the CTP framework. To see this, we neglect the finite density corrections in $\Sigma_{\ell}^{<,>}$implying that we set $f_{\ell}=$ $\bar{f}_{\ell}=f_{\phi}=\bar{f}_{\phi} \equiv 0$ in the propagators of the $T$ and $\bar{T}$ type. Then, in order to see which process a certain contribution in the collision term corresponds to, we cut through the propagators of the $<,>$ type, while leaving the propagators of the $T, \bar{T}$ type intact. We can then identify the terms in Eq. (44) that involve $\Psi_{N i j}^{T, \bar{T}}$ and $S_{N 1}^{<,>}$with decays and inverse decays. In the Feynman diagram representation of $\mathcal{F}_{\ell}$, that is given in Figure 1, this corresponds to the cut indicated by the dashed double line, which gives the interference term between the tree-level decay amplitudes and the wave-function corrections to the decay amplitudes. The terms in Eq. (44) that involve $\Psi_{N i j}^{<,>}$and $S_{N 1}^{T, \bar{T}}$ correspond to scatterings. Since $S_{N 1}^{T}$ and $S_{N 1}^{\bar{T}}$ are added together, precisely the pole contribution, when $N_{1}$ is on-shell, is isolated from the scatterings, which takes the effect of RIS subtraction. In the Feynman diagram in Figure 1 this corresponds to the cut indicated by the solid double lines. It represents the the squared amplitudes of the 


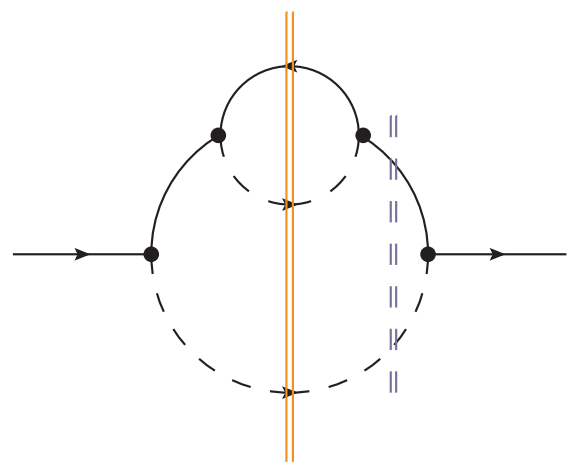

Figure 1: Diagrammatic representation of the lepton-number violating contribution to $\mathcal{Z}_{\ell}^{\mathrm{wf}<,>}$. The solid lines with arrows represent the lepton $\ell$, the solid lines without arrows the neutrinos $N_{1,2}$ and the dashed lines with arrows the Higgs boson $\phi$. The solid (light grey/orange) double line represents the cut that corresponds to the subtracted real intermediate states when finite density corrections to $C P$ violation are neglected. The dashed (dark-grey/blue) double line represents the cut that corresponds to decays and inverse decays when finite density corrections are neglected.

scatterings of leptons and Higgs bosons through $N_{1}$ and $N_{2}$ as well as the interference terms between these, which are crucial for the cancellation of $C P$-violating effects in equilibrium. Within the full CTP framework, $\&_{N i j}^{T, \bar{T}}$ and the $T, \bar{T}$-type propagators also acquire contributions from real particles in the plasma, such that the clear separation in decay and scattering processes through the cuts in Figure 1 no longer persists.

In order to see the role of the violation of time reversal symmetry, we note that for the vanishing of $\mathcal{K}_{i j}$, we make use of the KMS relation in the form of

$$
\mathrm{i} S_{N i}^{>}(k) \mathrm{i} S_{\ell}^{<}\left(k^{\prime}\right) \mathrm{i} \Delta_{\phi}^{<}\left(k^{\prime \prime}\right)=\mathrm{i} S_{N i}^{<}(k) \mathrm{i} S_{\ell}^{>}\left(k^{\prime}\right) \mathrm{i} \Delta_{\phi}^{>}\left(k^{\prime \prime}\right)
$$

for momenta satisfying $k=k^{\prime}+k^{\prime \prime}$. This equation is valid not only in thermal equilibrium, but it is also another way of expressing that the process $N_{i} \rightarrow \ell \phi$ and its inverse $\ell \phi \rightarrow N_{i}$ occur at the same rate, and that the same holds true for $N_{i} \rightarrow \bar{\ell} \phi^{*}$ and $\bar{\ell} \phi^{*} \rightarrow N_{i}$. Hence, the above discussion does not only show that no $C P$ asymmetry is generated in equilibrium, but more generally that no asymmetry is generated in situations that are symmetric with respect to time reversal, as it is of course expected by the CPT theorem.

\subsection{CP Source}

We now derive the $C P$-violating source within the lepton collision term, that arises due to deviations of $N_{1}$ from equilibrium and a violation of the KMS relations. First, we note that because of Eq. (6) we can identify

$$
\mathrm{i} \delta S_{N 1}^{>}=\mathrm{i} \delta S_{N 1}^{<}=\mathrm{i} \delta S_{N 1}^{T}=\mathrm{i} \delta S_{N 1}^{\bar{T}} \equiv \mathrm{i} \delta S_{N 1},
$$


where i $\delta S_{N 1}^{>,<, T, \bar{T}}$ denotes the difference between the non-equilibrium neutrino propagators and their equilibrium versions. Using this the $C P$-source collision term (38) reads

$$
\begin{aligned}
& \mathcal{C}_{\ell}^{\mathrm{wf}}(\mathbf{k})=\int \frac{d k_{0}}{2 \pi} \frac{d^{4} k^{\prime}}{(2 \pi)^{4}} \frac{d^{4} k^{\prime \prime}}{(2 \pi)^{4}}(2 \pi)^{4} \delta^{4}\left(k-k^{\prime}-k^{\prime \prime}\right)
\end{aligned}
$$

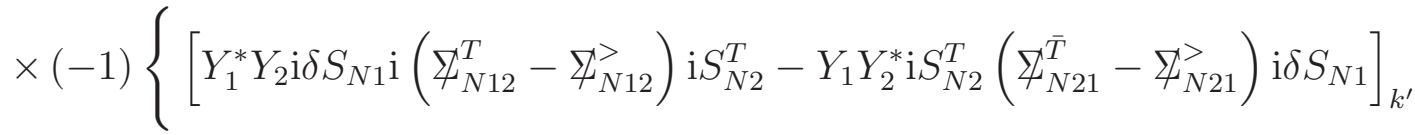

$$
\begin{aligned}
& \times \mathrm{i} \Delta_{\phi}^{<}\left(-k^{\prime \prime}\right) \mathrm{i} S_{\ell}^{<}(k) \\
& +\left[Y_{1}^{*} Y_{2} \mathrm{i} \delta S_{N 1} \mathrm{i}\left(\sharp_{N 12}^{\bar{T}}-\sharp_{N 12}^{<}\right) \mathrm{i} S_{N 2}^{T}-Y_{1} Y_{2}^{*} \mathrm{i} S_{N 2}^{T}\left(\sharp_{N 21}^{T}-\sharp_{N 21}^{<}\right) \mathrm{i} \delta S_{N 1}\right]_{k^{\prime}} \\
& \left.\times \mathrm{i} \Delta_{\phi}^{>}\left(-k^{\prime \prime}\right) \mathrm{i} S_{\ell}^{>}(k)\right\} .
\end{aligned}
$$

In this expression, the terms involving the dispersive parts $\Psi_{N i j}^{\operatorname{disp} T, \bar{T}}$ of $\Psi_{N i j}^{T, \bar{T}}$ cancel. To see this, first note that $i \Psi_{N i j}^{\text {disp } T}=-\mathrm{i} \Psi_{N i j}^{\text {disp } \bar{T}}$. Then, since $\delta S_{N 1}\left(k_{0}, \mathbf{k}\right)=\delta S_{N 1}\left(-k_{0}, \mathbf{k}\right)$ and since for equilibrium distributions $\mathrm{i} \Delta_{\phi}^{<}\left(k_{0}^{\prime}, \mathbf{k}^{\prime}\right) \mathrm{i} S_{\ell}^{<}\left(k_{0}^{\prime}, \mathbf{k}^{\prime}\right)=\mathrm{i} \Delta_{\phi}^{>}\left(-k_{0}^{\prime}, \mathbf{k}^{\prime}\right) \mathrm{i} S_{\ell}^{>}\left(-k_{0}^{\prime}, \mathbf{k}^{\prime}\right)$, the cancellation occurs when performing the integrals. We can therefore make the replacements

$$
\Psi_{i j}^{T, \bar{T}}-\Psi_{i j}^{>} \rightarrow \frac{1}{2}\left(\Psi_{i j}^{<}-\Psi_{i j}^{>}\right), \quad \Psi_{i j}^{T, \bar{T}}-\Psi_{i j}^{<} \rightarrow-\frac{1}{2}\left(\Psi_{i j}^{<}-\Psi_{i j}^{>}\right),
$$

and write the wave-function correction to the lepton collision integral as

$$
\begin{aligned}
\int \frac{d^{3} k}{(2 \pi)^{3}} \mathcal{C}_{\ell}^{\mathrm{wf}}(\mathbf{k})=\int \frac{d^{4} k}{(2 \pi)^{4}} \frac{d^{4} k^{\prime}}{(2 \pi)^{4}} \frac{d^{4} k^{\prime \prime}}{(2 \pi)^{4}}(2 \pi)^{4} \delta^{4}\left(k-k^{\prime}-k^{\prime \prime}\right) \\
\times \operatorname{tr}\left[\left(-Y_{1}^{*} Y_{2} \mathrm{i} \delta S_{N 1} \frac{\mathrm{i}}{2}\left(\psi_{N 12}^{<}-\Psi_{N 12}^{>}\right) \mathrm{i} S_{N 2}^{T}+Y_{1} Y_{2}^{*} \mathrm{i} S_{N 2}^{T} \frac{\mathrm{i}}{2}\left(\Psi_{N 21}^{<}-\Psi_{N 21}^{>}\right) \mathrm{i} \delta S_{N 1}\right)_{k^{\prime}}\right. \\
\left.\times\left(\mathrm{i} \Delta_{\phi}^{<}\left(-k^{\prime \prime}\right) \mathrm{i} S_{\ell}^{<}(k)-\mathrm{i} \Delta_{\phi}^{>}\left(-k^{\prime \prime}\right) \mathrm{i} S_{\ell}^{>}(k)\right)\right] .
\end{aligned}
$$

The spectral self-energy is

$$
\begin{aligned}
\mathrm{i} \Psi_{N i j}^{<}(k)-\mathrm{i} \&_{N i j}^{>}(k)= & -\int \frac{d^{3} k^{\prime}}{(2 \pi)^{3} 2\left|\mathbf{k}^{\prime}\right|^{2}} \frac{d^{3} k^{\prime \prime}}{(2 \pi)^{3} 2\left|\mathbf{k}^{\prime \prime}\right|^{2}}(2 \pi)^{4} \delta^{4}\left(k-k^{\prime}-k^{\prime \prime}\right) \\
& \times \operatorname{sign}\left(k_{0}\right)\left[Y_{i} Y_{j}^{*} \not k^{\prime} P_{\mathrm{R}}+Y_{i}^{*} Y_{j} \not k^{\prime} P_{\mathrm{L}}\right] \times\left[1-f_{\ell}^{\mathrm{eq}}\left(\mathbf{k}^{\prime}\right)+f_{\phi}^{\mathrm{eq}}\left(\mathbf{k}^{\prime \prime}\right)\right] .
\end{aligned}
$$

In further simplifying $\mathcal{C}_{\ell}^{\text {wf }}$, we obtain another factor of $\operatorname{sign}\left(k_{0}\right)$ from

$$
\begin{aligned}
& \mathrm{i} \Delta_{\phi}^{<}\left(-k^{\prime \prime}\right) \mathrm{i} S_{\ell}^{<}(k)-\left.\mathrm{i} \Delta_{\phi}^{>}\left(-k^{\prime \prime}\right) \mathrm{i} S_{\ell}^{>}(k)\right|_{\operatorname{sign}\left(k_{0}\right)=-\operatorname{sign}\left(k_{0}^{\prime \prime}\right)} \\
& =-P_{\mathrm{L}} \not k P_{\mathrm{R}}(2 \pi)^{2} \delta\left(k^{2}\right) \delta\left(k^{\prime \prime 2}\right) \operatorname{sign}\left(k_{0}\right)\left(1-f_{\ell}^{\mathrm{eq}}(\mathbf{k})+f_{\phi}^{\mathrm{eq}}\left(\mathbf{k}^{\prime \prime}\right)\right) .
\end{aligned}
$$


Using this result in Eq. (52), the tree-level propagators, and substituting after performing the Dirac trace the definition (33), we obtain our final result for the $C P$-violating wavefunction correction to the lepton collision term:

$$
\begin{aligned}
S^{\mathrm{wf}}= & \int \frac{d^{3} k}{(2 \pi)^{3}} \mathcal{C}_{\ell}^{\mathrm{wf}}(\mathbf{k}) \\
= & \int \frac{d^{3} k}{(2 \pi)^{3} 2|\mathbf{k}|} \frac{d^{3} k^{\prime}}{(2 \pi)^{3} 2 \sqrt{\mathbf{k}^{\prime 2}+M_{1}^{2}}} \frac{d^{3} k^{\prime \prime}}{(2 \pi)^{3} 2\left|\mathbf{k}^{\prime \prime}\right|}(2 \pi)^{4} \delta^{4}\left(k^{\prime}-k-k^{\prime \prime}\right) \\
& \times \frac{M_{1} M_{2}}{{k^{\prime}}^{2}-M_{2}^{2}} 2 \operatorname{Im}\left[Y_{1}^{2} Y_{2}^{* 2}\right] 2 k_{\mu} \Sigma_{N}^{\mu}\left(k^{\prime}\right) \delta f_{N}\left(\mathbf{k}^{\prime}\right)\left(1-f_{\ell}^{\mathrm{eq}}(\mathbf{k})+f_{\phi}^{\mathrm{eq}}\left(\mathbf{k}^{\prime \prime}\right)\right) \\
= & 4 \operatorname{Im}\left[Y_{1}^{2} Y_{2}^{* 2}\right] \frac{M_{1} M_{2}}{M_{1}^{2}-M_{2}^{2}} \int \frac{d^{3} k^{\prime}}{(2 \pi)^{3} 2 \sqrt{\mathbf{k}^{\prime 2}+M_{1}^{2}}} \delta f_{N}\left(\mathbf{k}^{\prime}\right) \frac{\Sigma_{N \mu}\left(\mathbf{k}^{\prime}\right) \Sigma_{N}^{\mu}\left(\mathbf{k}^{\prime}\right)}{g_{w}}
\end{aligned}
$$

Note that at zero temperature, we find

$$
\left.\Sigma_{N}^{\mu}(k)\right|_{T=0}=g_{w} \frac{k^{\mu}}{16 \pi} .
$$

Then, by comparison with the tree-level lepton collision term (20), we reproduce the zero-temperature result for the decay asymmetry

$$
\varepsilon_{T=0}^{\mathrm{wf}}=\frac{\Gamma_{N 1 \rightarrow \ell H}-\Gamma_{N 1 \rightarrow \bar{\ell} H^{\dagger}}}{\Gamma_{N 1 \rightarrow \ell H}+\Gamma_{N 1 \rightarrow \bar{\ell} H^{\dagger}}}=\frac{1}{8 \pi} \frac{\operatorname{Im}\left[Y_{1}^{2} Y_{2}^{* 2}\right]}{\left|Y_{1}\right|^{2}} \frac{M_{1} M_{2}}{M_{1}^{2}-M_{2}^{2}},
$$

where $\Gamma$ denotes the decay rate of the process indicated in the subscript. On the other hand, if finite-density effects are kept for the initial and final states but not in the self-energy loop, then in the final expression of Eq. (55) one substitutes

$$
\frac{\Sigma_{N \mu}\left(\mathbf{k}^{\prime}\right) \Sigma_{N}^{\mu}\left(\mathbf{k}^{\prime}\right)}{g_{w}} \rightarrow \frac{k^{\prime \mu} \Sigma_{N \mu}\left(\mathbf{k}^{\prime}\right)}{16 \pi} .
$$

\section{Vertex Contribution to the $C P$ Asymmetry}

\subsection{Vertex Correction to $\mathcal{C}_{\ell}$}

The vertex correction contribution to the $C P$ asymmetry arises from the two-loop selfenergy diagram for the lepton propagator (see Figure 2):

$$
\begin{aligned}
\mathrm{i} \Psi_{\ell}^{\mathrm{v} a b}(k)= & -c d Y_{i}^{* 2} Y_{j}^{2} \int \frac{d^{4} p}{(2 \pi)^{4}} \frac{d^{4} q}{(2 \pi)^{4}} P_{\mathrm{R}} \mathrm{i} S_{N i}^{a c}(-p) C\left[P_{\mathrm{L}} \mathrm{i} S_{\ell}^{d c}(p+k+q) P_{\mathrm{R}}\right]^{t} C^{\dagger} \\
& \times \mathrm{i} S_{N j}^{d b}(-q) P_{\mathrm{L}} \mathrm{i} \Delta_{\phi}^{d a}(-p-k) \mathrm{i} \Delta_{\phi}^{b c}(-q-k) .
\end{aligned}
$$

Here, the transposition $t$ acts on the Dirac indices, and $i, j$ and the CTP indices $c, d= \pm$ are summed over. The charge conjugation arises as a consequence of lepton number violation and the reversed flow of the internal lepton in the self-energy diagram. 
We again consider the case where $M_{2} \gg M_{1}$ in order to reduce the number of terms to be considered by one half. Furthermore, we neglect the diagonal $(i=j)$ contributions to the self-energy since they do not contribute to the $C P$ asymmetry. This can be easily verified along the lines of the calculation presented in this Section. With these simplifications, we obtain

$$
\begin{aligned}
& \mathrm{i} \sum_{\ell}^{\mathrm{v}>}(k)=-\int \frac{d^{4} p}{(2 \pi)^{4}} \frac{d^{4} q}{(2 \pi)^{4}} \\
& \times\left\{Y _ { 1 } ^ { * 2 } Y _ { 2 } ^ { 2 } \left[\mathrm{i} S_{N 1}^{>}(-p) C\left[P_{\mathrm{L}} \mathrm{i} S_{\ell}^{T}(p+k+q) P_{\mathrm{R}}\right]^{t} C^{\dagger} \mathrm{i} S_{N 2}^{T}(-q) \mathrm{i} \Delta_{\phi}^{<}(-p-k) \mathrm{i} \Delta_{\phi}^{T}(-q-k)\right.\right. \\
& \left.\quad-\mathrm{i} S_{N 1}^{\bar{T}}(-p) C\left[P_{\mathrm{L}} \mathrm{i} S_{\ell}^{<}(p+k+q) P_{\mathrm{R}}\right]^{t} C^{\dagger} \mathrm{i} S_{N 2}^{T}(-q) \mathrm{i} \Delta_{\phi}^{<}(-p-k) \mathrm{i} \Delta_{\phi}^{<}(-q-k)\right] \\
& +Y_{1}^{2} Y_{2}^{* 2}\left[-\mathrm{i} S_{N 2}^{\bar{T}}(-p) C\left[P_{\mathrm{L}} \mathrm{i} S_{\ell}^{<}(p+k+q) P_{\mathrm{R}}\right]^{t} C^{\dagger} \mathrm{i} S_{N 1}^{T}(-q) \mathrm{i} \Delta_{\phi}^{<}(-p-k) \mathrm{i} \Delta_{\phi}^{<}(-q-k)\right. \\
& \left.\left.\quad+\mathrm{i} S_{N 2}^{\bar{T}}(-p) C\left[P_{\mathrm{L}} \mathrm{i} S_{\ell}^{\bar{T}}(p+k+q) P_{\mathrm{R}}\right]^{t} C^{\dagger} \mathrm{i} S_{N 1}^{>}(-q) \mathrm{i} \Delta_{\phi}^{\bar{T}}(-p-k) \mathrm{i} \Delta_{\phi}^{<}(-q-k)\right]\right\} .
\end{aligned}
$$

The expression for $\mathrm{i}_{\ell}^{\mathrm{v}<}(k)$ is obtained through interchange of the CTP indices $T \leftrightarrow \bar{T}$ and $\langle\leftrightarrow\rangle$.

\subsection{KMS and Real Intermediate State Subtraction}

Before proceeding with the calculation of the $C P$ source, we again show that this contribution leads to a vanishing asymmetry in equilibrium, which in particular means that the KMS relation, $\mathrm{i}_{\ell}^{\mathrm{v}>}(k)+\mathrm{e}^{\beta k_{0}} \mathrm{i} \mathbb{Z}_{\ell}^{\mathrm{v}<}(k)=0$, must hold when equilibrium distributions are inserted. To see explicitly how this arises, we consider the contributions $\propto Y_{1}^{* 2} Y_{2}^{2}$. First, we define

$$
\mathrm{i} Z_{\ell}^{\mathrm{v}>}(k)+\mathrm{e}^{\beta k_{0}} \mathrm{i} \not_{\ell}^{\mathrm{v}<}(k)=\int \frac{d^{4} p}{(2 \pi)^{4}} \frac{d^{4} q}{(2 \pi)^{4}} \mathcal{J}(k, p, q)
$$

and note that by virtue of the KMS relation

$$
\begin{aligned}
\mathcal{J}(k, p, q)= & Y_{1}^{* 2} Y_{2}^{2}\left[\mathrm{i} S_{N 1}^{T}(-p)+\mathrm{i} S_{N 1}^{\bar{T}}(-p)\right] C\left[P_{\mathrm{L}} \mathrm{i} S_{\ell}^{<}(p+k+q) P_{\mathrm{R}}\right]^{t} C^{\dagger} \mathrm{i} S_{N 2}^{T}(-q) \\
& \times \mathrm{i} \Delta_{\phi}^{<}(-p-k) \mathrm{i} \Delta_{\phi}^{<}(-q-k) \\
- & Y_{1}^{* 2} Y_{2}^{2} \mathrm{i} S_{N 1}^{>}(-p) C\left[P_{\mathrm{L}} \mathrm{i} S_{\ell}^{T}(p+k+q) P_{\mathrm{R}} \Delta_{\phi}^{T}(-q-k)\right. \\
& \left.+P_{\mathrm{L}} \mathrm{i} S_{\ell}^{\bar{T}}(p+k+q) P_{\mathrm{R}} \Delta_{\phi}^{\bar{T}}(-q-k)\right]^{t} C^{\dagger} \mathrm{i} S_{N 2}^{T}(-q) \mathrm{i} \Delta_{\phi}^{<}(-p-k) .
\end{aligned}
$$

Further simplification is achieved when deriving from the explicit expressions (4) and (8) that for $\operatorname{sign}\left(r_{0}\right)=\operatorname{sign}\left(s_{0}\right)$,

$$
\mathrm{i} S_{\ell}^{T}(r) \mathrm{i} \Delta_{\phi}^{T}(s)+\mathrm{i} S_{\ell}^{\bar{T}}(r) \mathrm{i} \Delta_{\phi}^{\bar{T}}(s)=\mathrm{i} S_{\ell}^{<}(r) \mathrm{i} \Delta_{\phi}^{<}(s)+\mathrm{i} S_{\ell}^{>}(r) \mathrm{i} \Delta_{\phi}^{>}(s) .
$$




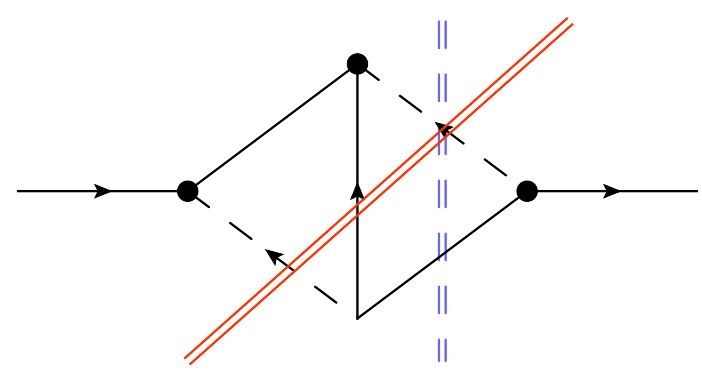

Figure 2: Diagrammatic representation of $\not_{\ell}^{\mathrm{v}<,>}$. The solid lines with arrows represent the lepton $\ell$, the solid lines without arrows the neutrinos $N_{1,2}$ and the dashed lines with arrows the Higgs boson $\phi$. The solid (light-grey/orange) double line represents the cut that corresponds to the subtracted real intermediate states when finite density corrections to $C P$ violation are neglected. The dashed (dark-grey/blue) double line represents the cut that corresponds to decays and inverse decays when finite density corrections are neglected.

We find

$$
\begin{aligned}
\mathcal{J}(k, p, q)= & Y_{1}^{* 2} Y_{2}^{2}\left[\mathrm{i} S_{N 1}^{<}(-p)+\mathrm{i} S_{N 1}^{>}(-p)\right] C\left[P_{\mathrm{L}} \mathrm{i} S_{\ell}^{<}(p+k+q) P_{\mathrm{R}}\right]^{t} C^{\dagger} \mathrm{i} S_{N 2}^{T}(-q) \\
& \times \mathrm{i} \Delta_{\phi}^{<}(-p-k) \mathrm{i} \Delta_{\phi}^{<}(-q-k) \\
- & Y_{1}^{* 2} Y_{2}^{2} \mathrm{i} S_{N 1}^{>}(-p) C\left[P_{\mathrm{L}} \mathrm{i} S_{\ell}^{<}(p+k+q) P_{\mathrm{R}} \Delta_{\phi}^{<}(-q-k)\right. \\
& \left.+P_{\mathrm{L}} \mathrm{i} S_{\ell}^{>}(p+k+q) P_{\mathrm{R}} \Delta_{\phi}^{>}(-q-k)\right]^{t} C^{\dagger} \mathrm{i} S_{N 2}^{T}(-q) \mathrm{i} \Delta_{\phi}^{<}(-p-k) \\
= & 0
\end{aligned}
$$

where for the last equality, we have again used the KMS relation. Likewise, one can show the vanishing of the terms $\propto Y_{1}^{2} Y_{2}^{* 2}$ in equilibrium.

Again, we can relate this verification of the KMS relation to the standard procedure of RIS subtraction. As in the wave-function contribution, at zero temperature the $<,>$ type Green functions correspond to cut propagators. The terms in (60) involving $S_{N 1}^{<,>}$ and $\Delta_{\phi}^{<,>}$thus correspond to the cut indicated by the dashed double line in Figure 2 that represents the interference between the tree-level and one-loop contribution to the $N_{1}$ decay amplitude. The terms involving $S_{N 1}^{T, \bar{T}}$ on the other hand correspond to $N_{1}$ mediated scatterings, as indicated by the solid cut line in Figure 2. Note that here the cut corresponds to the interference between $s$-channel and $t$-channel diagrams, while the central cuts through the wave-function correction yield the squares of the $s$-channel and $t$-channel contributions to the scatterings. This complementarity is crucial for the separate cancellation of the asymmetries from the wave function and the vertex correction in equilibrium. 


\section{$5.3 \quad C P$ Source}

We now extract the $C P$ source in the lepton collision term using the same simplifications as in the calculation of the wave-function correction. In particular, the deviation of $N_{1}$ from equilibrium is parametrised again by $\delta S_{N 1}$. We obtain

$$
\begin{aligned}
\mathcal{C}_{\ell}^{\mathrm{v}}(\mathbf{k})= & \int \frac{d k_{0}}{2 \pi} \operatorname{tr}\left[\mathrm{i} \xi_{\ell}^{\mathrm{v}>}(k) \mathrm{i} S_{\ell}^{<}(k)-\mathrm{i} Y_{\ell}^{\mathrm{v}<}(k) \mathrm{i} S_{\ell}^{>}(k)\right] \\
=\int \frac{d k_{0}}{2 \pi} & \frac{d^{4} p}{(2 \pi)^{4}} \frac{d^{4} q}{(2 \pi)^{4}} \times \operatorname{tr}\left[\left\{-Y_{1}^{* 2} Y_{2}^{2} \mathrm{i} \delta S_{N 1}(-p) C\left[\mathrm{i} S_{\ell}^{T}(p+k+q) \mathrm{i} \Delta_{\phi}^{T}(-q-k)\right.\right.\right. \\
& \left.-\mathrm{i} S_{\ell}^{<}(p+k+q) \mathrm{i} \Delta_{\phi}^{<}(-q-k)\right]^{t} C^{\dagger} \mathrm{i} S_{N 2}^{T}(-q) \mathrm{i} \Delta_{\phi}^{<}(-p-k) \\
+ & Y_{1}^{2} Y_{2}^{* 2} \mathrm{i} S_{N 2}^{T}(-p) C\left[\mathrm{i} S_{\ell}^{\bar{T}}(p+k+q) \mathrm{i} \Delta_{\phi}^{\bar{T}}(-q-k)\right. \\
& \left.\left.-\mathrm{i} S_{\ell}^{<}(p+k+q) \mathrm{i} \Delta_{\phi}^{<}(-q-k)\right]^{t} C^{\dagger} \mathrm{i} \delta S_{N 1}^{T}(-q) \mathrm{i} \Delta_{\phi}^{<}(-p-k)\right\} \mathrm{i} S_{\ell}^{<}(k) \\
- & -Y_{1}^{2} Y_{2}^{* 2} \mathrm{i} S_{N 2}^{T}(-p) C\left[\mathrm{i} S_{\ell}^{T}(p+k+q) \mathrm{i} \Delta_{\phi}^{T}(-q-k)\right. \\
& \left.-\mathrm{i} S_{\ell}^{>}(p+k+q) \mathrm{i} \Delta_{\phi}^{>}(-q-k)\right]^{t} C^{\dagger} \mathrm{i} \delta S_{N 1}^{T}(-q) \mathrm{i} \Delta_{\phi}^{>}(-p-k) \\
+ & Y_{1}^{* 2} Y_{2}^{2} \mathrm{i} \delta S_{N 1}(-p) C\left[\mathrm{i} S_{\ell}^{\bar{T}}(p+k+q) \mathrm{i} \Delta_{\phi}^{\bar{T}}(-q-k)\right. \\
& \left.\left.\left.-\mathrm{i} S_{\ell}^{>}(p+k+q) \mathrm{i} \Delta_{\phi}^{>}(-q-k)\right]^{t} C^{\dagger} \mathrm{i} S_{N 2}^{T}(-q) \mathrm{i} \Delta_{\phi}^{>}(-p-k)\right\} \mathrm{i} S_{\ell}^{>}(k)\right],
\end{aligned}
$$

where $\mathrm{i} S_{N 2}^{\bar{T}}=-\mathrm{i} S_{N 2}^{T}$ for off-shell neutrinos $N_{2}$ has been used. Inspection of the Dirac structure reveals that $S_{N 1,2}$ only contribute through terms proportional to $M_{1,2}$, which is familiar from the standard calculation of the decay asymmetry [1]. Finally, similar to the replacement (51) we can substitute here

$$
\begin{aligned}
& S_{\ell}^{T, \bar{T}} \Delta_{\phi}^{T, \bar{T}}-S_{\ell}^{>} \Delta_{\phi}^{>} \rightarrow+\frac{1}{2}\left(S_{\ell}^{<} \Delta_{\phi}^{<}-S_{\ell}^{>} \Delta_{\phi}^{>}\right), \\
& S_{\ell}^{T, \bar{T}} \Delta_{\phi}^{T, \bar{T}}-S_{\ell}^{<} \Delta_{\phi}^{<} \rightarrow-\frac{1}{2}\left(S_{\ell}^{<} \Delta_{\phi}^{<}-S_{\ell}^{>} \Delta_{\phi}^{>}\right)
\end{aligned}
$$

under the integrals.

The expression (65) closely resembles the result for the wave-function contribution, except that i $S_{N 2}^{T}$ now depends on the loop momentum $q$. In the limit where $M_{2} \gg M_{1}$, the $N_{2}$ propagator can be expanded in powers of $\tilde{k}^{2} / M_{2}^{2}$ where $\tilde{k}=(p, k)$, showing that in this limit the vertex correction is just one half of the self-energy contribution, where the factor one half arises since there is no summation over $\mathrm{SU}(2)$ indices within the loop.

Inserting the explicit expressions for the lepton and Higgs boson Green functions, we obtain our final result for the vertex correction to the $C P$ asymmetry. To this end we 
introduce

$$
\begin{aligned}
\Gamma_{\mu}\left(k, p^{\prime \prime}, M_{2}\right)= & \int \frac{d^{3} k^{\prime}}{(2 \pi)^{3} 2\left|\mathbf{k}^{\prime}\right|} \frac{d^{3} k^{\prime \prime}}{(2 \pi)^{3} 2\left|\mathbf{k}^{\prime \prime}\right|}(2 \pi)^{4} \delta^{4}\left(k-k^{\prime}-k^{\prime \prime}\right) k_{\mu}^{\prime} \frac{M_{1} M_{2}}{\left(k^{\prime}-p^{\prime \prime}\right)^{2}-M_{2}^{2}} \\
& \times\left[1-f_{\ell}\left(\mathbf{k}^{\prime}\right)+f_{\phi}\left(\mathbf{k}^{\prime \prime}\right)\right] .
\end{aligned}
$$

This has to be integrated over the initial and final state phase space in the collision term, such that we additionally define

$$
\begin{aligned}
V\left(k, M_{2}\right)= & \int \frac{d^{3} p^{\prime}}{(2 \pi)^{3} 2\left|\mathbf{p}^{\prime}\right|} \frac{d^{3} p^{\prime \prime}}{(2 \pi)^{3} 2\left|\mathbf{p}^{\prime \prime}\right|}(2 \pi)^{4} \delta^{4}\left(k-p^{\prime}-p^{\prime \prime}\right) p^{\prime \mu} \Gamma_{\mu}\left(k, p^{\prime \prime}, M_{2}\right) \\
& \times\left[1-f_{\ell}\left(\mathbf{p}^{\prime}\right)+f_{\phi}\left(\mathbf{p}^{\prime \prime}\right)\right] .
\end{aligned}
$$

In terms of these quantities, the lepton collision term can be expressed as

$$
S^{\mathrm{v}}=\int \frac{d^{3} p^{\prime}}{(2 \pi)^{3}} \mathcal{C}_{\ell}^{\mathrm{v}}\left(\mathbf{p}^{\prime}\right)=4 \operatorname{Im}\left[Y_{1}^{2} Y_{2}^{* 2}\right] \int \frac{d^{3} k}{(2 \pi)^{3}} \delta f_{N 1}(\mathbf{k}) V\left(k, M_{2}\right) .
$$

\subsection{Source term in the strongly hierarchical limit}

For the strongly hierarchical case, $M_{2} \gg M_{1}$, which we will focus on for the numerical analysis, the vertex contribution is simply one half of the self energy contribution. To see this, we note that in the limit $M_{2} \gg M_{1}$,

$$
\frac{M_{1} M_{2}}{\left(k^{\prime}-p^{\prime \prime}\right)^{2}-M_{2}^{2}} \rightarrow-\frac{M_{1}}{M_{2}}
$$

in Eq. (67), which implies

$$
\Gamma_{\mu}\left(k, p^{\prime \prime}, M_{2}\right)=-\frac{M_{1}}{M_{2}} \frac{\Sigma_{\mu}(k)}{g_{W}} \quad \text { and } \quad V\left(k, M_{2}\right)=-\frac{M_{1}}{M_{2}} \frac{\Sigma_{N \mu}\left(\mathbf{k}^{\prime}\right) \Sigma_{N}^{\mu}\left(\mathbf{k}^{\prime}\right)}{g_{w}^{2}} .
$$

Inserting this into (69) and adding the wave-function contribution (55) the source term for the lepton asymmetry in Eq. (31) is given by

$$
S_{M_{2} \gg M_{1}}=\frac{3}{2} \times 4 \operatorname{Im}\left[Y_{1}^{2} Y_{2}^{* 2}\right]\left(-\frac{M_{1}}{M_{2}}\right) \int \frac{d^{3} k^{\prime}}{(2 \pi)^{3} 2 \sqrt{\mathbf{k}^{\prime 2}+M_{1}^{2}}} \delta f_{N}\left(\mathbf{k}^{\prime}\right) \frac{\Sigma_{N \mu}\left(\mathbf{k}^{\prime}\right) \Sigma_{N}^{\mu}\left(\mathbf{k}^{\prime}\right)}{g_{w}}
$$

with $\Sigma_{N \mu}\left(\mathbf{k}^{\prime}\right)$ given in Eq. (33).

\section{Numerical Estimates of Finite Density Effects}

In this Section we solve the kinetic equation for the final lepton asymmetry with $C P$ violating collision terms derived in Sections $3-5$. We compare our results with all finite density effects included to the case where these effects are included in the initial and final state phase-space distributions, but not in the quantum corrections. 


\subsection{Effective $\boldsymbol{C P}$-violating parameter}

To begin with, however, we present a rough estimate for the total magnitude of $C P$ violation in the lepton collision term. This estimate is provided through an effective $C P$-violating parameter, which we define as:

$$
\varepsilon_{\mathrm{eff}} \equiv-\frac{\frac{d}{d \eta}\left(n_{\ell}-\bar{n}_{\ell}\right)-W}{\frac{d}{d \eta} n_{N 1}},
$$

where $n_{\ell, N 1}=\int \frac{d^{3} k}{(2 \pi)^{3}} f_{\ell, N 1}(\mathbf{k})$ are the comoving number densities of lepton and neutrino. The tree-level (washout) contribution $W$, see Eq. (37), is subtracted from this definition, so that the numerator involves only the $C P$ source, which is comprised of self-energy and vertex type corrections to the lepton collision term, given by Eqs. $(55,69)$. The $C P$-violating parameter thus also breaks into the corresponding two parts. For the self-energy part we get by using Eqs. (34), (55):

$$
\varepsilon_{\mathrm{eff}}^{\mathrm{wf}}=\varepsilon_{T=0}^{\mathrm{wf}} \frac{16 \pi}{g_{w}} \frac{\int d|\mathbf{k}| \frac{|\mathbf{k}|^{2}}{k_{0}} \sum_{N 1}^{\mu}(k) \Sigma_{N 1 \mu}(k) \delta f_{N 1}(\mathbf{k})}{\int d|\mathbf{k}| \frac{|\mathbf{k}|^{2}}{k_{0}} k_{\mu} \Sigma_{N 1}^{\mu}(k) \delta f_{N 1}(\mathbf{k})},
$$

where $\varepsilon_{T=0}^{\mathrm{wf}}$ is the $C P$-violating parameter in the vacuum, given by Eq. (57). We see right away, that Eq. (74) indeed reduces to $\varepsilon_{T=0}^{\mathrm{wf}}$ in the limit where the finite density effects in the loop are neglected in which case one factor of $\Sigma_{N}^{\mu}(k)$ in the numerator is replaced by $g_{w} k^{\mu} /(16 \pi)$. We will not compute the vertex correction part here in full generality, but we note that in the strongly hierarchical case, where $M_{2} \gg M_{1}$, the vertex $C P$-source reduces to one-half of the self energy $C P$-source, as shown in the previous Section. The same is then obviously true for the corresponding $C P$-violating parameter $\varepsilon_{\mathrm{eff}}^{\mathrm{v}}$, so that the ratio $\varepsilon_{\text {eff }} / \varepsilon_{T=0}$ is the same for the total and the wave-function contribution alone.

To compute $\varepsilon_{\text {eff }}^{\mathrm{wf}}$ requires the knowledge of the neutrino distribution function $f_{N 1}(\mathbf{k})$ for each instant of time (or temperature in the expanding Universe), which is obtained by solving the tree-level Boltzmann equation (34). Before doing this we make an estimate for $\varepsilon_{\mathrm{eff}}^{\mathrm{wf}}$ by factoring out the nontrivial time-dependence in Eq. (74). This can be achieved by assuming that the decaying heavy neutrino $N_{1}$ remains in kinetic equilibrium and making an ansatz with a pseudo-chemical potential $\mu_{N 1}$ :

$$
f_{N 1}(\mathbf{k})=\frac{1}{\exp \left[\beta\left(\sqrt{\mathbf{k}^{2}+M_{1}^{2}}-\mu_{N 1}\right)\right]+1} .
$$

For small deviations from thermal equilibrium, this can be expanded in linear order of $\mu_{N 1}$ as

$$
\delta f_{N 1}(\mathbf{k})=f_{N 1}^{\mathrm{eq}}(\mathbf{k})\left(1-f_{N 1}^{\mathrm{eq}}(\mathbf{k})\right) \frac{\mu_{N 1}}{T} .
$$

Using this ansatz the pseudo-chemical potential $\mu_{N 1}$ involving the time dependence cancels out in the ratio in Eq. (74), and we get

$$
\varepsilon_{\mathrm{eff}}^{\mathrm{wf}}=\varepsilon_{T=0}^{\mathrm{wf}} \frac{16 \pi}{g_{w}} \frac{\int d|\mathbf{k}| \frac{|\mathbf{k}|^{2}}{k_{0}} \Sigma_{N 1}^{\mu}(k) \Sigma_{N 1 \mu}(k) f_{N 1}^{\mathrm{eq}}(\mathbf{k})\left(1-f_{N 1}^{\mathrm{eq}}(\mathbf{k})\right)}{\int d|\mathbf{k}| \frac{|\mathbf{k}|^{2}}{k_{0}} k_{\mu} \Sigma_{N 1}^{\mu}(k) f_{N 1}^{\mathrm{eq}}(\mathbf{k})\left(1-f_{N 1}^{\mathrm{eq}}(\mathbf{k})\right)} .
$$


To evaluate this expression, we see that the phase space integrals in $\Sigma_{N 1}^{\mu}(k)$, Eq. (33), can be computed to give

$$
\begin{aligned}
& \Sigma_{N}^{0}(k)=\frac{g_{w} T^{2}}{8 \pi|\mathbf{k}|} I_{1}\left(\frac{k_{0}}{T}, \frac{|\mathbf{k}|}{T}\right) \\
& \Sigma_{N}^{i}(k)=\frac{g_{w} T^{2}}{8 \pi|\mathbf{k}|}\left(\frac{k_{0}}{|\mathbf{k}|} I_{1}\left(\frac{k_{0}}{T}, \frac{|\mathbf{k}|}{T}\right)-\frac{M_{1}^{2}}{2|\mathbf{k}| T} I_{0}\left(\frac{k_{0}}{T}, \frac{|\mathbf{k}|}{T}\right)\right) \frac{k^{i}}{|\mathbf{k}|},
\end{aligned}
$$

with

$$
I_{n}\left(y_{0}, y\right) \equiv \int_{\frac{1}{2}\left(y_{0}-y\right)}^{\frac{1}{2}\left(y_{0}+y\right)} d x x^{n}\left(1-\frac{1}{\mathrm{e}^{x}+1}+\frac{1}{\mathrm{e}^{y_{0}-x}-1}\right)
$$

which have analytical expressions

$$
\begin{aligned}
I_{0}\left(y_{0}, y\right)= & \ln \left(\frac{e^{y_{0}+y}-1}{e^{y_{0}-y}-1}\right)-y, \\
I_{1}\left(y_{0}, y\right)= & \frac{1}{2}\left(y_{0}+y\right) \ln \left(\frac{1+e^{\frac{y_{0}+y}{2}}}{1-e^{\frac{-y_{0}+y}{2}}}\right)-\frac{1}{2}\left(y_{0}-y\right) \ln \left(\frac{1+e^{\frac{y_{0}-y}{2}}}{1-e^{\frac{-y_{0}-y}{2}}}\right) \\
& +\operatorname{Li}_{2}\left(-e^{\frac{y_{0}+y}{2}}\right)+\operatorname{Li}_{2}\left(e^{\frac{-y_{0}-y}{2}}\right)-\operatorname{Li}_{2}\left(-e^{\frac{y_{0}-y}{2}}\right)-\operatorname{Li}_{2}\left(e^{\frac{-y_{0}+y}{2}}\right),
\end{aligned}
$$

where $\mathrm{Li}_{2}$ denotes dilogarithm. The remaining one-dimensional momentum integrations in Eq. (77) can then easily be computed numerically. We plot the resulting ratio $\varepsilon_{\mathrm{eff}}^{\mathrm{wf}} / \varepsilon_{T=0}^{\mathrm{wf}}$ (equal to $\varepsilon_{\text {eff }} / \varepsilon_{T=0}$ in the strongly hierarchical limit) as a function of $z=M_{1} / T$ in Figure 3. We find that the medium effects tend to enhance the effective asymmetry, in particular, the Bose enhancement of the Higgs particle dominates over the Fermi suppression of the lepton.

\subsection{Numerical solution of the Boltzmann equations}

We now scrutinise how the enhancement of the asymmetry is reflected in the solution of the Boltzmann equations (30), (31). For our numerical analysis we consider the asymmetries in the limit of strong hierarchy: $M_{2} \gg M_{1}$, when the total $C P$ source in Eq. (31) is given by Eq. (72). The vector $\Sigma_{N}^{\mu}$ can be evaluated according to Eqs. (78) when substituting for $T$ the "comoving" temperature $T_{\text {com }}=a(\eta) T$. The washout term on the right-hand side of Eq. (31) has been given in Eq. (37).

In the parametric region of strong washout, the neutrino density and also the lepton asymmetry will eventually become independent of the initial conditions, while this is not the case in the weak-washout domain. When dropping the assumption that the right-handed neutrinos are in equilibrium $[16,17]$, we can integrate the equations for 


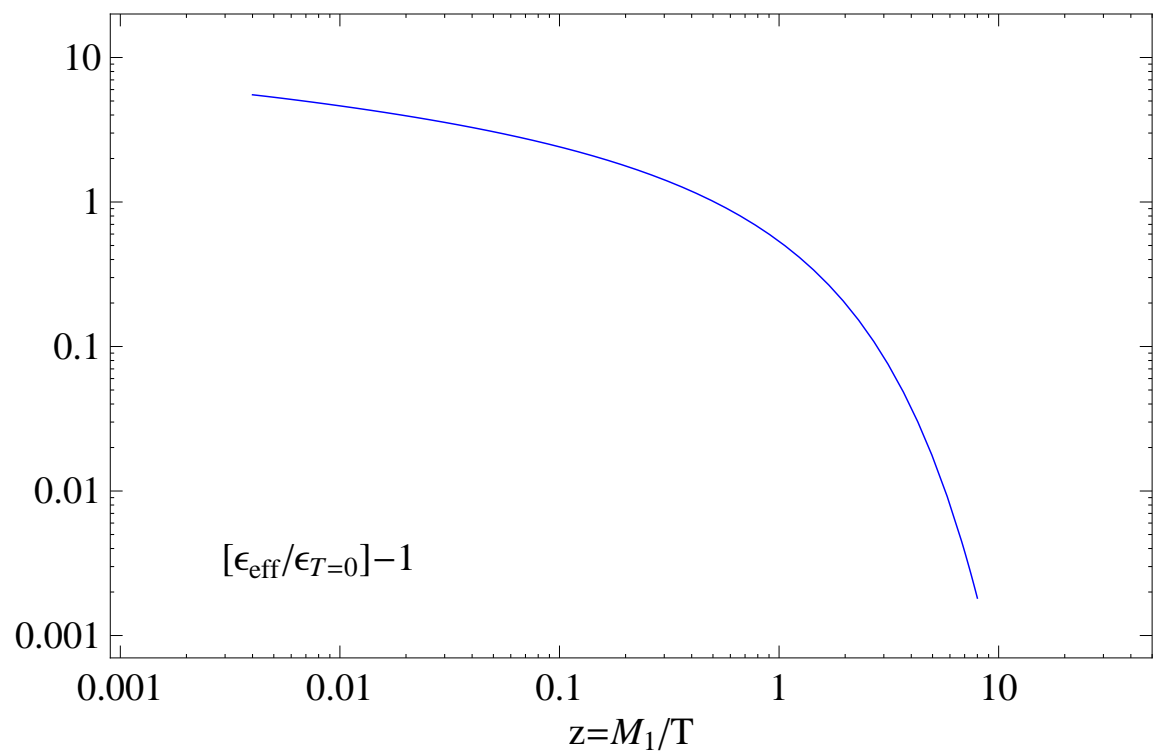

Figure 3: The ratio of the $C P$-violating parameter when taking effects of finite density in the loop into account over the $C P$-violating parameter at $T=0$.

the comoving modes $\mathbf{k}_{\mathrm{com}}$ of the neutrino number distribution separately, which is the method that we pursue here. Thus, to solve the time-evolution equation for the lepton asymmetry, we first calculate the out-of-equilibrium neutrino distribution by solving Eq. (30) with the decay and inverse decay term $D\left(\mathbf{k}_{\mathrm{com}}\right)$ given by Eq. (34). This solution is then inserted into the source term. The expression (34) carries dependence on conformal time $\eta$ through the dependence on $k_{\text {com } 0}=\sqrt{\mathbf{k}_{\text {com }}^{2}+a_{\mathrm{R}}^{2} \eta^{2} M_{1}^{2}}$, and the dependence of $\Sigma_{N}^{\mu}\left(k_{\text {com }}\right)$ and $f_{N 1}^{\text {eq }}\left(\mathbf{k}_{\text {com }}\right)$ on $k_{\text {com } 0}$. Thus a numerical solution of Eq. (30) for the out-of-equilibrium neutrino distribution is necessary.

In Figure 4, we show solutions to the Boltzmann equations in the intermediate regime between weak and strong washout. What is shown is the asymmetry summed over spin and $\mathrm{SU}(2)$ degree of freedoms and normalized to the entropy density,

$$
Y_{\ell}=2 g_{w} \frac{n_{\mathrm{ph}, \ell}-\bar{n}_{\mathrm{ph}, \ell}}{s}=2 g_{w} \frac{n_{\mathrm{com}, \ell}-\bar{n}_{\mathrm{com}, \ell}}{\frac{2 \pi^{2}}{45} g_{\star} T_{\mathrm{com}}^{3}},
$$

where the last form is convenient since we calculate directly the comoving particle densities. We assume an effective number of relativistic degrees of freedom according to the Standard Model $\left(g_{*}=106.75\right)$. In the Figure, we provide results both with (solid lines) and without (dashed lines) the additional finite density enhancement in the loops. We choose the parameters $M_{1}=10^{13} \mathrm{GeV}, M_{2}=10^{15} \mathrm{GeV},\left|Y_{1}\right|=2 \times 10^{-2},\left|Y_{2}\right|=10^{-1}$ and a maximal $C P$ phase such that $\operatorname{Im}\left[Y_{1}^{2} Y_{2}^{* 2}\right]$ is purely imaginary. Besides, we consider two different initial conditions, namely vanishing (light grey/red lines) and thermal (dark grey/blue lines) initial distributions for $N_{1}$. Recall also that the parameter $z=M_{1} / T$ 


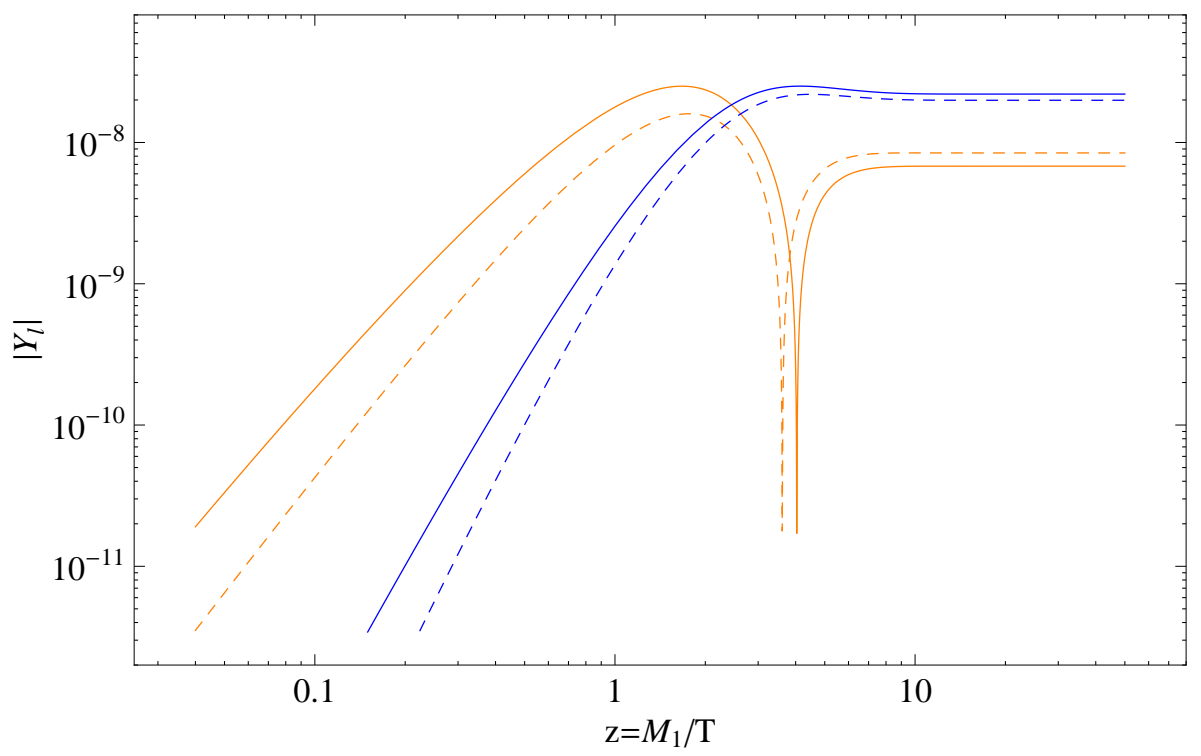

Figure 4: The absolute value of lepton-to-entropy ratio $Y_{\ell}$ over $z=M_{1} / T$. The parameters are $M_{1}=10^{13} \mathrm{GeV}, M_{2}=10^{15} \mathrm{GeV}, Y_{1}=2 \times 10^{-2}, Y_{2}=10^{-1}$ and a maximal $C P$ phase. Both, thermal initial conditions (dark grey/blue) and vanishing initial conditions (light grey/red) for $N_{1}$ are chosen. The solid lines correspond to solutions where finite density corrections in the loop are taken into account, the dotted lines to solutions where these are omitted.

is proportional to $\eta$. We observe from the Figure that there is a sizable effect of order $(10-20) \%$ from the finite density terms in the loops surviving at late times, since the asymmetry is partly produced at early times, when $M_{1}$ is not much larger than $T$ and quantum statistical corrections are of importance. This situation no longer persists at larger coupling, $Y_{1}=5 \times 10^{-2}$, corresponding to strong washout. We display the result in the upper panel of Figure 5. Here the final asymmetry is determined at late times when $M_{1} \gg T$, such that quantum statistical factors have no sizable impact.

Finally, we consider a scenario of weak washout (here we choose $Y_{1}=10^{-2}$ ). The resulting asymmetries are displayed in the lower panel of Figure 5. In the case of vanishing initial conditions for $N_{1}$, we find the impact of the finite-density effects in the loop in this scenario quite drastic: When they are taken into account, the asymmetry produced at early times when the $N_{1}$-abundance is below its equilibrium value becomes larger, due to the Bose enhancement of the Higgs particles. This "wrong-sign" asymmetry is then too large to be cancelled by the "right-sign" asymmetry produced at later times, when the $N_{1}$-abundance is above its equilibrium value. Not only the temperature dependence of the washout, but also the temperature-dependence of the effective $C P$-violating parameter is crucial for determining the final lepton asymmetry. This feature does not occur when the loop enhancement is neglected, since in that case, the effective $C P$ asymmetry 

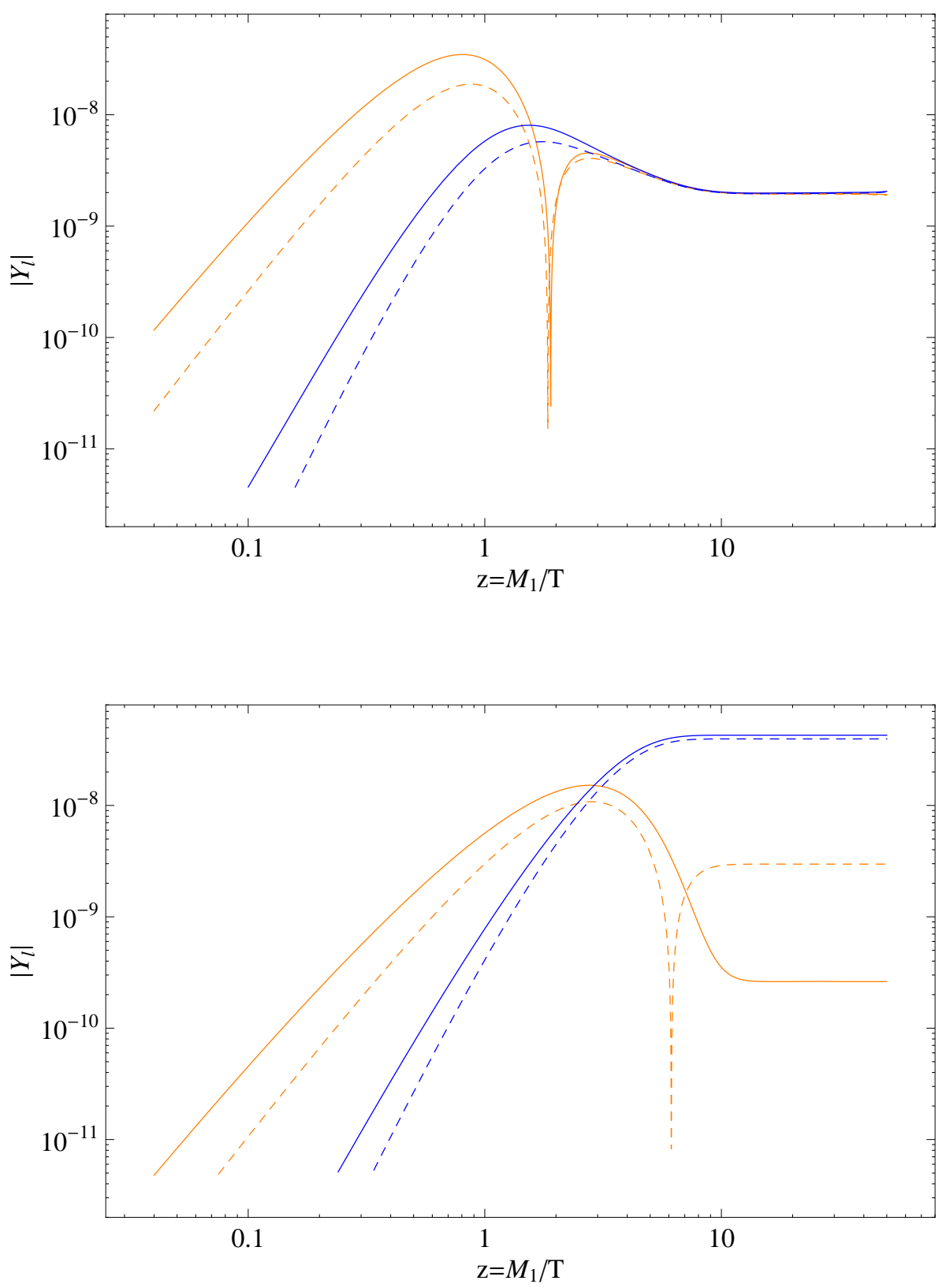

Figure 5: The absolute value of lepton-to-entropy ratio $Y_{\ell}$ over $z=M_{1} / T$. The parameters are $M_{1}=10^{13} \mathrm{GeV}, M_{2}=10^{15} \mathrm{GeV}, Y_{1}=5 \times 10^{-2}, Y_{2}=10^{-1}$ and a maximal $C P$ phase in the upper panel (strong washout) and $Y_{1}=10^{-2}, Y_{2}=10^{-1}$ in the lower panel (weak washout). Both, thermal initial conditions (dark grey/blue) and vanishing initial conditions (light grey/red) for $N_{1}$ are chosen. The solid lines correspond to solutions where finite density corrections in the loop are taken into account, the dotted lines to solutions where these are omitted. 
is temperature independent and the resulting asymmetry is always of the "right-sign". Therefore, neglecting the finite density effects in the loop in weak washout scenarios is in general not justifiable and may under certain circumstances even lead to an error in the predicted sign of the asymmetry.

\section{Conclusions}

We presented a full derivation and numerical solution of the kinetic equations describing leptogenesis in the CTP framework. The results extend existing approaches since all quantum statistical factors are accounted for systematically for the first time in a realistic model of leptogenesis. We have put particular emphasis on establishing a connection to the conventional Boltzmann approach and elucidated how RIS subtraction is realised in the CTP formalism. The numerical analysis shows that finite density corrections do not play a significant role in the strong-washout regime while they may have a sizable impact in weak-washout scenarios.

The detailed analysis of the simple model of leptogenesis presented in this work should be seen as a first step toward applications of the CTP formalism to situations where the Boltzmann framework needs to be modified. One may think of flavour effects $[23,24,25,26]$, the inclusion of additional thermal effects [15] or resonant leptogenesis $[27,21,28,24]$ in the limit of degenerate right-handed neutrino masses. Examples of approaches to these more advanced problems using the CTP formalism may already be found the literature $[10,11,29]$. However, so far there has been no complete derivation and solution of the equations describing the standard scenario of leptogenesis within the CTP framework. The present work may be viewed as a self-consistent leading-order approximation in this framework, which serves as a starting point for investigating further corrections and variations.

\section{Note added}

While this paper was in writing, Ref. [30] appeared which also studies modifications of an effective $C P$-violating parameter in a realistic leptogenesis model due to finite-density effects in loops.

\section{Acknowledgements}

This work is supported by the Gottfried Wilhelm Leibniz programme of the Deutsche Forschungsgemeinschaft and by the Schweizer Nationalfonds. PS would like to thank RWTH Aachen University for hospitality.

\section{References}

[1] M. Fukugita and T. Yanagida, "Baryogenesis Without Grand Unification," Phys. Lett. B 174 (1986) 45. 
[2] S. Davidson, E. Nardi and Y. Nir, "Leptogenesis," Phys. Rept. 466 (2008) 105 [arXiv:0802.2962 [hep-ph]].

[3] D. V. Nanopoulos and S. Weinberg, "Mechanisms For Cosmological Baryon Production," Phys. Rev. D 20 (1979) 2484.

[4] E. W. Kolb and S. Wolfram, "Baryon Number Generation In The Early Universe," Nucl. Phys. B 172 (1980) 224 [Erratum-ibid. B 195 (1982) 542].

[5] A. D. Sakharov, "Violation of $C P$ Invariance, $C$ Asymmetry, and Baryon Asymmetry of the Universe," Pisma Zh. Eksp. Teor. Fiz. 5 (1967) 32 [JETP Lett. 5 (1967 SOPUA,34,392-393.1991 UFNAA,161,61-64.1991) 24].

[6] J. S. Schwinger, "Brownian motion of a quantum oscillator," J. Math. Phys. 2 (1961) 407.

[7] L. V. Keldysh, "Diagram technique for nonequilibrium processes," Zh. Eksp. Teor. Fiz. 47 (1964) 1515 [Sov. Phys. JETP 20 (1965) 1018].

[8] E. Calzetta and B. L. Hu, "Nonequilibrium Quantum Fields: Closed Time Path Effective Action, Wigner Function and Boltzmann Equation," Phys. Rev. D 37 (1988) 2878.

[9] W. Buchmüller and S. Fredenhagen, "Quantum mechanics of baryogenesis," Phys. Lett. B 483 (2000) 217 [arXiv:hep-ph/0004145].

[10] A. De Simone and A. Riotto, "Quantum Boltzmann Equations and Leptogenesis," JCAP 0708 (2007) 002 [arXiv:hep-ph/0703175].

[11] A. Anisimov, W. Buchmüller, M. Drewes and S. Mendizabal, "Quantum Interference in a Thermal Bath," arXiv:1001.3856 [hep-ph].

[12] M. Garny, A. Hohenegger, A. Kartavtsev and M. Lindner, "Systematic approach to leptogenesis in nonequilibrium QFT: vertex contribution to the CP-violating parameter," Phys. Rev. D 80 (2009) 125027 [arXiv:0909.1559 [hep-ph]].

[13] M. Garny, A. Hohenegger, A. Kartavtsev and M. Lindner, "Systematic approach to leptogenesis in nonequilibrium QFT: self-energy contribution to the CP-violating parameter," arXiv:0911.4122 [hep-ph].

[14] L. Covi, N. Rius, E. Roulet and F. Vissani, "Finite temperature effects on CP violating asymmetries," Phys. Rev. D 57 (1998) 93 [arXiv:hep-ph/9704366].

[15] G. F. Giudice, A. Notari, M. Raidal, A. Riotto and A. Strumia, "Towards a complete theory of thermal leptogenesis in the SM and MSSM," Nucl. Phys. B 685 (2004) 89 [arXiv:hep-ph/0310123]. 
[16] A. Basboll and S. Hannestad, "Decay of heavy Majorana neutrinos using the full Boltzmann equation including its implications for leptogenesis," JCAP 0701 (2007) 003 [arXiv:hep-ph/0609025].

[17] F. Hahn-Woernle, M. Plumacher and Y. Y. Y. Wong, "Full Boltzmann equations for leptogenesis including scattering," JCAP 0908 (2009) 028 [arXiv:0907.0205 [hep$\mathrm{ph}]]$.

[18] T. Prokopec, M. G. Schmidt and S. Weinstock, "Transport equations for chiral fermions to order h-bar and electroweak baryogenesis," Annals Phys. 314 (2004) 208 [arXiv:hep-ph/0312110].

[19] T. Prokopec, M. G. Schmidt and S. Weinstock, "Transport equations for chiral fermions to order h-bar and electroweak baryogenesis. II," Annals Phys. 314 (2004) 267 [arXiv:hep-ph/0406140].

[20] B. Garbrecht and T. Konstandin, "Separation of Equilibration Time-Scales in the Gradient Expansion," Phys. Rev. D 79 (2009) 085003 [arXiv:0810.4016 [hep-ph]].

[21] A. Pilaftsis, "CP violation and baryogenesis due to heavy Majorana neutrinos," Phys. Rev. D 56 (1997) 5431 [arXiv:hep-ph/9707235].

[22] W. Buchmüller, P. Di Bari and M. Plümacher, "Leptogenesis for pedestrians," Annals Phys. 315 (2005) 305 [arXiv:hep-ph/0401240].

[23] T. Endoh, T. Morozumi and Z. h. Xiong, "Primordial lepton family asymmetries in seesaw model," Prog. Theor. Phys. 111 (2004) 123 [arXiv:hep-ph/0308276].

[24] A. Pilaftsis and T. E. J. Underwood, "Electroweak-scale resonant leptogenesis," Phys. Rev. D 72 (2005) 113001 [arXiv:hep-ph/0506107].

[25] A. Abada, S. Davidson, F. X. Josse-Michaux, M. Losada and A. Riotto, "Flavour Issues in Leptogenesis," JCAP 0604, 004 (2006) [arXiv:hep-ph/0601083].

[26] E. Nardi, Y. Nir, E. Roulet and J. Racker, "The importance of flavor in leptogenesis," JHEP 0601, 164 (2006) [arXiv:hep-ph/0601084].

[27] M. Flanz, E. A. Paschos and U. Sarkar, "Baryogenesis from a lepton asymmetric universe," Phys. Lett. B 345 (1995) 248 [Erratum-ibid. B 382 (1996) 447] [arXiv:hep-ph/9411366].

[28] A. Pilaftsis and T. E. J. Underwood, "Resonant leptogenesis," Nucl. Phys. B 692 (2004) 303 [arXiv:hep-ph/0309342].

[29] V. Cirigliano, C. Lee, M. J. Ramsey-Musolf and S. Tulin, "Flavored Quantum Boltzmann Equations," arXiv:0912.3523 [hep-ph].

[30] M. Garny, A. Hohenegger and A. Kartavtsev, "Medium corrections to the CPviolating parameter in leptogenesis," arXiv:1002.0331 [hep-ph]. 